\title{
12. RARE EARTH ELEMENT COMPOSITION OF ANHYDRITE: IMPLICATIONS FOR DEPOSITION AND MOBILITY WITHIN THE ACTIVE TAG HYDROTHERMAL MOUND ${ }^{1}$
}

\author{
Susan E. Humphris ${ }^{2}$
}

\begin{abstract}
Rare earth elements (REEs) of 39 anhydrite samples from different textural settings and from three different areas on the active TAG hydrothermal mound have been analyzed to examine the geochemical evolution of the circulating fluid. All of the chondrite-normalized REE patterns of anhydrite are characterized by positive Eu anomalies that range from 2 to 18 and varying levels of light rare earth (LREE) enrichment with $\mathrm{Nd}_{\mathrm{N}} / \mathrm{Yb}_{\mathrm{N}}$ values from 5 to 25 . No systematic variations in the REE patterns are observed with depth or with textural setting.

In the TAG-1 area, the samples fall into two groups when normalized to the composition of the end-member black smoker hydrothermal fluid. The first group shows relatively flat REE patterns $\left(\mathrm{Nd}_{\mathrm{FL}} / \mathrm{Yb}_{\mathrm{FL}}\right.$ of $\left.0.8-2.8\right)$ with only a small or no Eu anomaly ranging from 0.8 to 1.5 . If it is assumed that the Group I anhydrites were derived from mixing of seawater with an endmember black smoker hydrothermal fluid, then this implies that, during the precipitation of this type of anhydrite from the circulating fluid, all of the REEs, including Eu, are taken up in proportions that reflect their relative distribution in the fluid. Consideration of the redox conditions of the hydrothermal fluid during mixing with either cold or conductively heated seawater indicates that both divalent and trivalent Eu may be present. Hence, a possible explanation for the flat REE patterns relative to hydrothermal fluid is that the trivalent Eu was not discriminated against during precipitation, and hence there was no fractionation of Eu relative to the other REEs. Mixing of a hydrothermal fluid with seawater would result in a decrease in temperature and an increase in $\mathrm{fO}_{2}$ in the mix relative to the hydrothermal fluid, both of which would result in more Eu being present as the trivalent ion. An alternative possibility that cannot be ruled out is that the Group I anhydrites precipitated from mixing of seawater with a hydrothermal fluid of different composition.

The second group is characterized by a distinct negative Eu anomaly ranging from 0.2 to 0.7 relative to the end-member hydrothermal fluid. This implies that during the precipitation of this anhydrite group, Eu is excluded relative to the other REEs, resulting in an increase in the concentration of Eu in the fluid while the concentrations of other REEs in the fluid decrease. There is an inverse correlation between Eu anomaly and the absolute REE concentrations in anhydrite suggesting that the Eu anomaly can be used as an indicator of the degree of evolution of the hydrothermal fluid. In addition, an increase in the LREE enrichment as the fluid evolves is also observed, which may be related to the greater stability of LREE chloride complexes in high temperature, low $\mathrm{pH}$ fluids.

In the TAG-2 area, the Eu anomalies in the anhydrite are more strongly developed than in the TAG-1 area, suggesting that they have formed from hydrothermal fluids that had previously mixed with seawater and precipitated anhydrite to a greater degree than those in the TAG-1 area.

The chondrite-normalized pattern of the one anhydrite sample from the TAG-5 area shows some distinct differences from the other samples analyzed. The absolute REE concentrations in this sample, with the exception of Eu, are the highest found in this study and the Eu anomaly, although still positive, is extremely small. Possible mechanisms to explain this REE pattern in these samples include mixing between a hydrothermal fluid that has undergone conductive cooling prior to mixing, or addition of REEs to the hydrothermal fluid prior to mixing with seawater by dissolution of anhydrite.

The REE data provide evidence for the entrainment of seawater and mixing with hydrothermal fluids down to at least 58.68 mbsf. The mixing process is complex and chaotic at all scales, and shows no systematic variations either laterally or vertically on the mound scale, or even within an individual vein.
\end{abstract}

\section{INTRODUCTION}

In September-November 1994, during Leg 158 of the Ocean Drilling Program (ODP), a series of holes was drilled through the surficial deposit and uppermost parts of the underlying stockwork at the TAG hydrothermal mound on the Mid-Atlantic Ridge, to investigate the subsurface nature of an active, volcanic-hosted hydrothermal system on a slow-spreading ridge. One of the most notable results from the drilling leg was the abundance of anhydrite, particularly within the upflow zone beneath the region of high-temperature activity (Humphris et al., 1995; Humphris, Herzig, Miller, et al.,1996). Although previous studies had documented anhydrite on the surface of the mound (Thompson et al., 1988; Tivey et al., 1995), and geochemical modeling of fluid compositions had predicted precipitation of an-

${ }^{1}$ Herzig, P.M., Humphris, S.E., Miller, D.J., and Zierenberg, R.A. (Eds.), 1998. Proc. ODP, Sci. Results, 158: College Station, TX (Ocean Drilling Program).

${ }^{2}$ Department of Geology and Geophysics, Woods Hole Oceanographic Institution, Woods Hole, MA 02543, U.S.A. shumphris@whoi.edu hydrite within the mound (Tivey et al., 1995), the extent of precipitation was unexpected, particularly because it is rare or absent in most land-based ophiolite massive sulfide deposits generally believed to be the ancient analogs of modern seafloor hydrothermal systems. Anhydrite exhibits retrograde solubility and will not precipitate from seawater until temperatures of $>150^{\circ} \mathrm{C}$ (at seafloor pressures) are attained; hence, its presence not only implies the maintenance of high temperatures within the upflow zone, but also the entrainment, and subsequent heating, of substantial amounts of seawater beneath the mound (Humphris et al., 1995; Tivey et al., 1995). Mixing of hydrothermal fluids and seawater has therefore played a critical role in the complex history of fluid flow within the mound and in the development of its internal structure.

The precipitation of anhydrite from fluids circulating and mixing within the mound provides the opportunity to investigate the extent and distribution of subsurface mixing and the evolution of the circulating fluid. Previous studies of the rare earth element (REE) distributions in hydrothermal fluids and surface samples (including sulfides, sulfates, and oxides) from the mound have indicated that anhydrite is the major phase that removes the REEs from solution at TAG

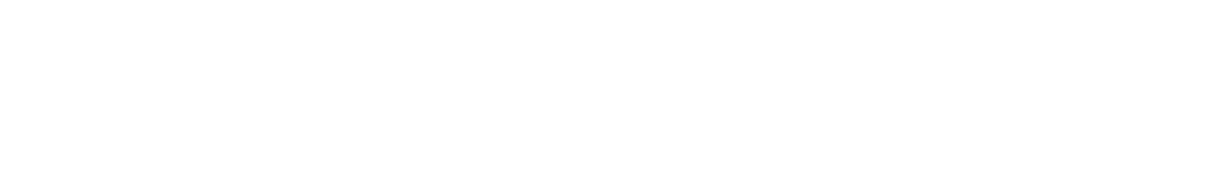


(Mitra et al., 1994; Mills and Elderfield, 1995). In addition, the REE geochemistry of basalts and seawater has been well characterized (e.g., Elderfield and Greaves, 1982; Saunders, 1984; Elderfield, 1988). This paper presents the results of a study of the distribution of the REEs in anhydrite in order to examine the geochemical evolution of the circulating fluids.

\section{SURFACE MORPHOLOGY OF THE ACTIVE TAG HYDROTHERMAL MOUND}

The geologic setting of the active TAG hydrothermal mound has recently been summarized by Rona et al. (1993b) and Humphris, Herzig, Miller, et al. (1996). Briefly, it is located within a $5 \mathrm{~km} \times 5$ $\mathrm{km}$ hydrothermal field that includes both sites of active high and lowtemperature venting and extensive relict seafloor deposits (Thompson et al., 1985; Rona et al., 1993a). The active TAG hydrothermal mound is located at a water depth of $3650 \mathrm{~m}$ at the base of the eastern wall of the median valley of the Mid-Atlantic Ridge at $26^{\circ} 08^{\prime} \mathrm{N}$, where two series of intersecting faults are postulated to provide permeable pathways for upflow of hydrothermal fluids (Kleinrock and Humphris, 1996; Kleinrock et al., 1996). The mound is built on crust that is at least 100,000 yr old, and it is thought to have been episodically active for at least the last 20,000 yr (Lalou et al., 1990; 1993).

Observations from submersibles and detailed high-resolution photographic studies prior to drilling documented the surface morphology of the mound (Thompson et al., 1988; Rona et al., 1993b; Tivey et al., 1995; Kleinrock et al., 1996; Humphris and Kleinrock, 1996). In addition, the geochemistry of both hydrothermal fluids and mineral precipitates collected from the mound have been described (Campbell et al., 1988; Thompson et al., 1988; Edmond et al., 1995; Tivey et al., 1995). The mound is about $200 \mathrm{~m}$ in diameter and comprises two distinct platforms, the surfaces of which are rough and composed of blocky massive sulfides and anhydrite. Hydrothermal fluids discharge as diffuse flow over much of the mound, and as more focused flow in two areas. High-temperature $\left(363^{\circ} \mathrm{C}\right)$ black smoker activity is confined to the upper platform, with the most vigorous and focused flow localized in a complex of large chalcopyrite-anhydriterich chimneys (over $10 \mathrm{~m}$ high) on top of a cone, 10-20 m high with a basal diameter of 20-30 m, the surface of which is a platy layer of massive chalcopyrite and marcasite with interspersed blocks of massive anhydrite. Lower temperature $\left(260^{\circ}-300^{\circ} \mathrm{C}\right)$ white smoker activity occurs in the "Kremlin" area on the southeast portion of the lower platform, where fluids emanate from small $(1-2 \mathrm{~m})$ spires composed dominantly of low-Fe sphalerite. Although the black and white smoker fluids have distinct chemistries, the white smoker fluid can be derived from the end-member black smoker fluid through processes of conductive cooling, mixing with entrained seawater, and precipitation and dissolution of various mineral phases within the mound (Edmond et al., 1995; Tivey et al., 1995).

\section{INTERNAL STRATIGRAPHY OF THE TAG ACTIVE MOUND}

The nature of the interior of the mound and the underlying stockwork down to $125 \mathrm{mbsf}$ was revealed during Leg 158 when seventeen holes comprising Site 957 were drilled in five major areas on the active TAG mound (Fig. 1). Breccias of various types dominate the sections, and several lithologic zones have been identified (Fig. 2; Humphris et al., 1995; Humphris, Herzig, Miller, et al., 1996). Massive sulfides are restricted to the upper few meters at each drilling location and reflect the formation of new hydrothermal precipitates. The upper $10-20 \mathrm{~m}$ of the mound is made up of pyrite breccias that consist of clasts of pyrite in a matrix of pyrite (with minor quartz and/ or anhydrite). At those sites near the complex of black smokers (i.e., the TAG-1 and TAG-5 areas), the pyrite breccias are underlain by an anhydrite-rich zone. Down to about $30 \mathrm{mbsf}$, this zone consists of pyrite-anhydrite breccias composed dominantly of clasts of pyrite in a matrix of $>10 \mathrm{vol} \%$ anhydrite. Below $30 \mathrm{mbsf}$ and down to the base of the anhydrite-rich zone at $45 \mathrm{mbsf}$, pyrite-silica-anhydrite breccias are present, which are composed of pyrite, quartz + pyrite, and silicified basalt clasts in a matrix of $>10 \mathrm{vol} \%$ anhydrite + quartz. Anhydrite veins are abundant in this zone, with complex veins up to $45 \mathrm{~cm}$ wide exhibiting several generations of precipitation and dissolution. The anhydrite-rich zone is underlain by pyrite-silica breccias composed of clasts similar to those in the zone above, but in a matrix of $>10 \mathrm{vol} \%$ quartz. Anhydrite veins are still common in this zone near the black smoker complex, and also occur in the pyrite-silica breccias at TAG-2. At TAG-1, TAG-4, and TAG-5, the pyrite-silica breccias grade into silicified wallrock breccias, which consist of clasts of basalt totally replaced by quartz, paragonite, pyrite, and a Ti-bearing phase in a matrix of quartz and pyrite. Below about $110 \mathrm{mbsf}$ at TAG1 , chloritized basalt breccias occur and consist of chloritized basalt fragments cemented by quartz and pyrite.

Anhydrite occurs in three textural settings in the TAG-1, TAG-2, and TAG-4 areas: in the matrix of breccias, filling vugs, and as veins. Examples of these are shown in Figures 3 to 5, which also document the locations of multiple samples taken from these sections. The veins are best developed in the TAG-1 area, although they are also present in cores from TAG-2 and TAG-5. Three types of veins have been distinguished by shipboard scientists (Humphris, Herzig, Miller, et al., 1996). Massive, crustiform, banded veins have individual growth bands from a few millimeters up to $1 \mathrm{~cm}$, with finegrained pyrite, chalcopyrite, and rare hematite commonly disseminated along them. The textures of these veins suggest that they result from sequential precipitation in open space (either a cavity or repeated fracture opening). Massive, granular anhydrite veins are not as common, and tend to occur as discrete bands within the large crustiform-banded veins. They are coarsely crystalline, usually lack welldefined growth bands, and are gray because of the presence of abundant, uniformly disseminated, fine-grained pyrite and chalcopyrite. The third type of vein is late-stage, anhydrite veins that cut across earlier anhydrite and sulfide breccias. They are typically narrow (up to $2 \mathrm{~cm}$ ) with bands of euhedral anhydrite crystals, and they lack disseminated sulfides.

The recovery of basaltic basement at TAG- 2 and TAG- 4 suggests that the upper portion of the stockwork zone may be only about 150 $\mathrm{m}$ in diameter, although the possibility that the basement rocks recovered at TAG-2 may be an overlying basaltic flow cannot be ruled out. However, a near-bottom magnetic survey over the TAG mound showed the presence of a magnetization low of about $100 \mathrm{~m}$ in diameter directly beneath the mound, which has been interpreted as the alteration pipe of the upflow zone (Tivey et al., 1993). This would therefore support the conclusion that the stockwork zone does not extend beyond the edges of the mound.

\section{SAMPLE SELECTION AND METHODS}

Anhydrite samples were selected from the TAG-1, TAG-2, and TAG-5 areas in order to obtain (1) a representative sampling of anhydrite both from the anhydrite-rich zone and from other depths at TAG-1 and TAG-5, and also of anhydrites from other lithologic zones at TAG-2, and (2) more detailed sampling of anhydrite from its different textural settings, including the matrix of breccias, vug fillings, different vein types, as well as different growth bands within individual veins. Brief descriptions are given in Table 1 . Most of the samples for the more detailed studies of different vein types and growth bands were taken from Hole $957 \mathrm{C}$ because core recovery in the anhydrite-rich zone down to 49 mbsf was particularly good (averaging 64\%), and, hence, the relations between samples could be 


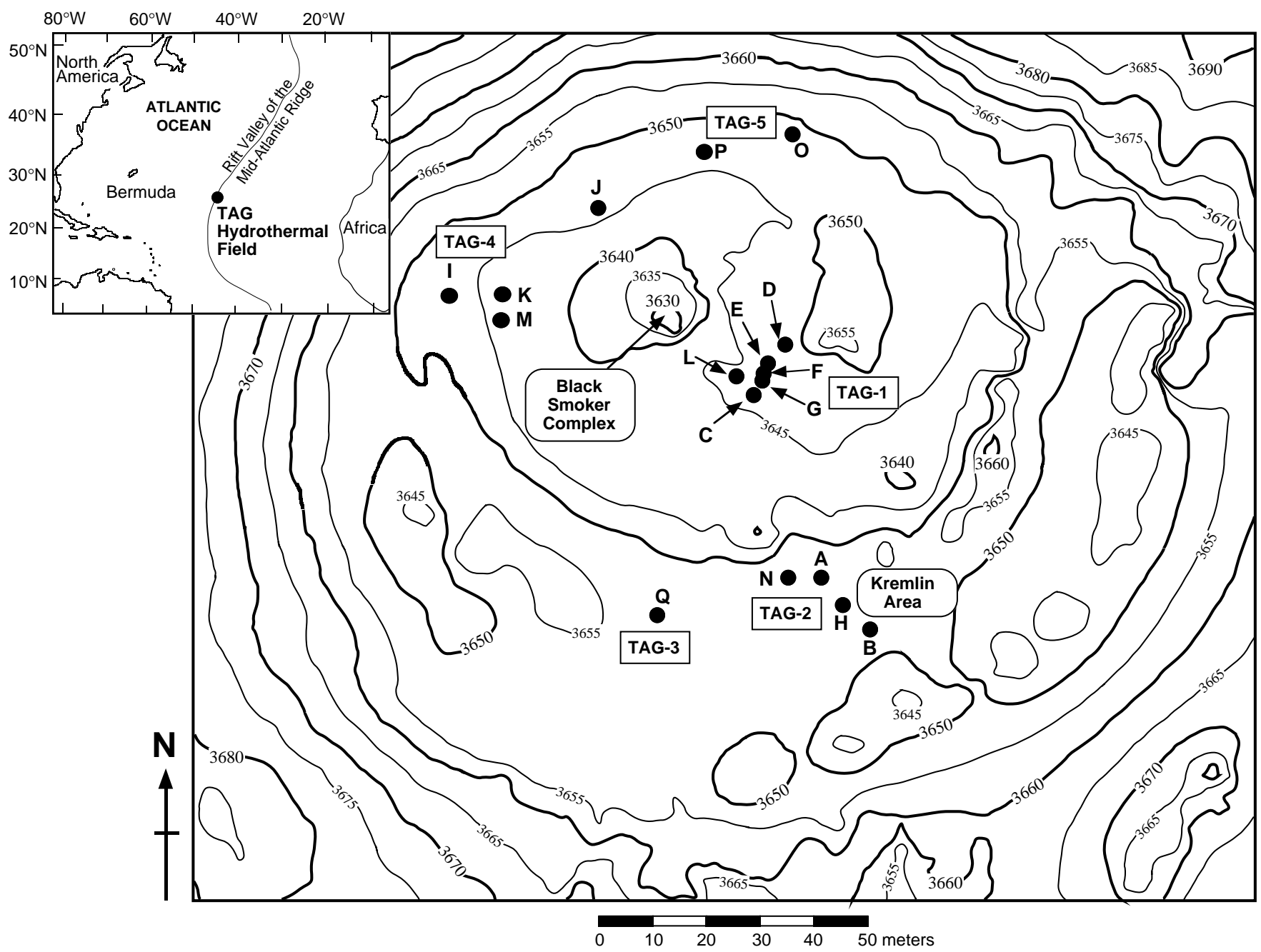

Figure 1. High-resolution bathymetry (5-m contour interval) of the TAG active mound, showing its overall structure and the locations of the holes drilled during Leg 158 (Humphris et al., 1995). Inset shows the location of the TAG hydrothermal field on the Mid-Atlantic Ridge.

better established. In particular, a number of samples were analyzed from different generations of vein filling in a $45-\mathrm{cm}$-thick vein in Section 957C-11N-1, as shown in Figure 4. Only one sample was obtained from TAG-5.

Anhydrite was removed from the samples by either hand-picking individual grains when the crystals were sufficiently large, chipping off small pieces with a chisel, or by drilling out areas using a Dremel tool. Every sample was then carefully hand-picked under a binocular microscope to separate anhydrite from any sulfides and hematite.

Rare earth element concentrations were determined by comparison with a set of standards using a Sciex Elan model 250 ICP-MS at the Washington State University GeoAnalytical Laboratory. Details of the dissolution and analytical methods are described in Knaack et al. (1991). Analytical precision for all the REEs is in the range of $1 \%-2 \%$.

\section{RESULTS}

The results of the REE analyses of anhydrite are presented in Table 2 by location and depth, together with the REE contents of TAG hydrothermal fluids and seawater (Mitra et al., 1994). Concentrations of REEs are generally in the range of 0.001-4 ppm, although the sample from TAG-5 exhibits concentrations up to about $6 \mathrm{ppm}$ for $\mathrm{Ce}$ and $\mathrm{Nd}$. These concentrations are about three orders of magnitude greater than those found in end-member black smoker hydrothermal fluids, and four to seven orders of magnitude greater than the REE concentrations in seawater. For comparative purposes, the concentration data have been normalized to the average REE concentrations for chondrites (Sun and McDonough, 1989). For reference, the chondrite-normalized REE patterns for black and white smoker fluids and seawater are presented in Figure 6. Because seawater plays a role in the precipitation of anhydrite in hydrothermal systems and exhibits a large negative $\mathrm{Ce}$ anomaly, $\mathrm{Nd}_{\mathrm{N}} / \mathrm{Yb}_{\mathrm{N}}$ is used to compare the slopes of the chondrite-normalized REE patterns.

\section{REE Variations Within and Between the TAG-1, TAG-2, and TAG-5 Areas}

The chondrite-normalized REE patterns for anhydrite from the TAG-1 area (Fig. 7) have been grouped by depth in order to present the large amount of data. All of the samples show light rare earth element (LREE - La to Gd) enrichment as demonstrated by $\mathrm{Nd}_{\mathrm{N}} / \mathrm{Yb}_{\mathrm{N}}$ values, which range from 5.1 to 24.9. In addition, all patterns show a marked positive $\mathrm{Eu}$ anomaly, with values of $\mathrm{Eu}_{\mathrm{N}} / \mathrm{Eu}^{*}{ }_{\mathrm{N}}\left(\right.$ where $\mathrm{Eu}^{*}{ }_{\mathrm{N}}$ is defined as the value of $\mathrm{Eu}_{\mathrm{N}}$ obtained by straight line interpolation between the plotted points for $\mathrm{Sm}_{\mathrm{N}}$ and $\mathrm{Gd}_{\mathrm{N}}$ ) generally in the range 2.1-9.8, although one sample shows a higher Eu anomaly with a Eu $/$ 


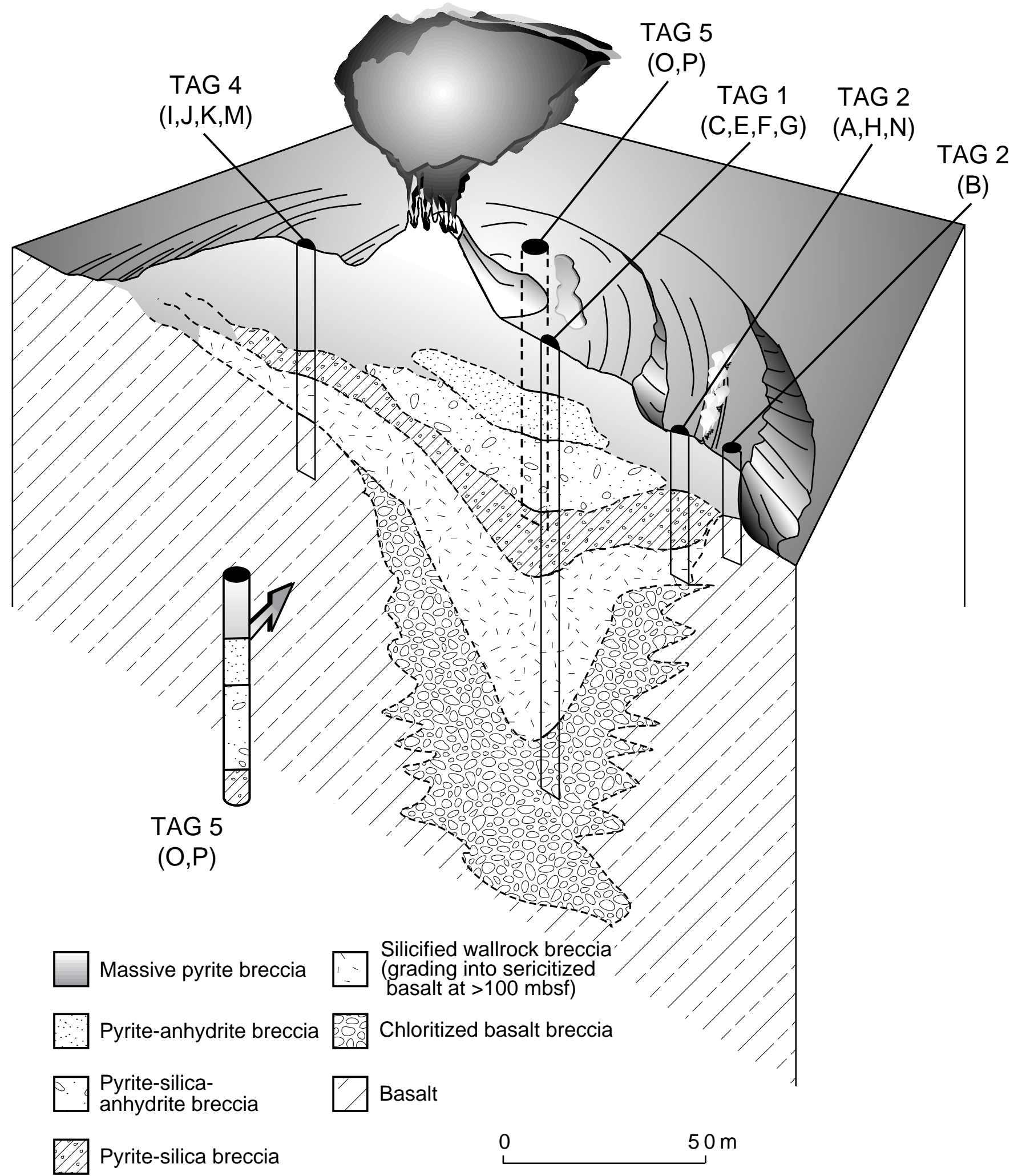

Figure 2. Sketch of the TAG active mound showing the generalized internal stratigraphy and lithologic zones defined by the results from drilling. Letters in parentheses refer to the drill holes at each area. (Modified from Humphris et al., 1995.) 


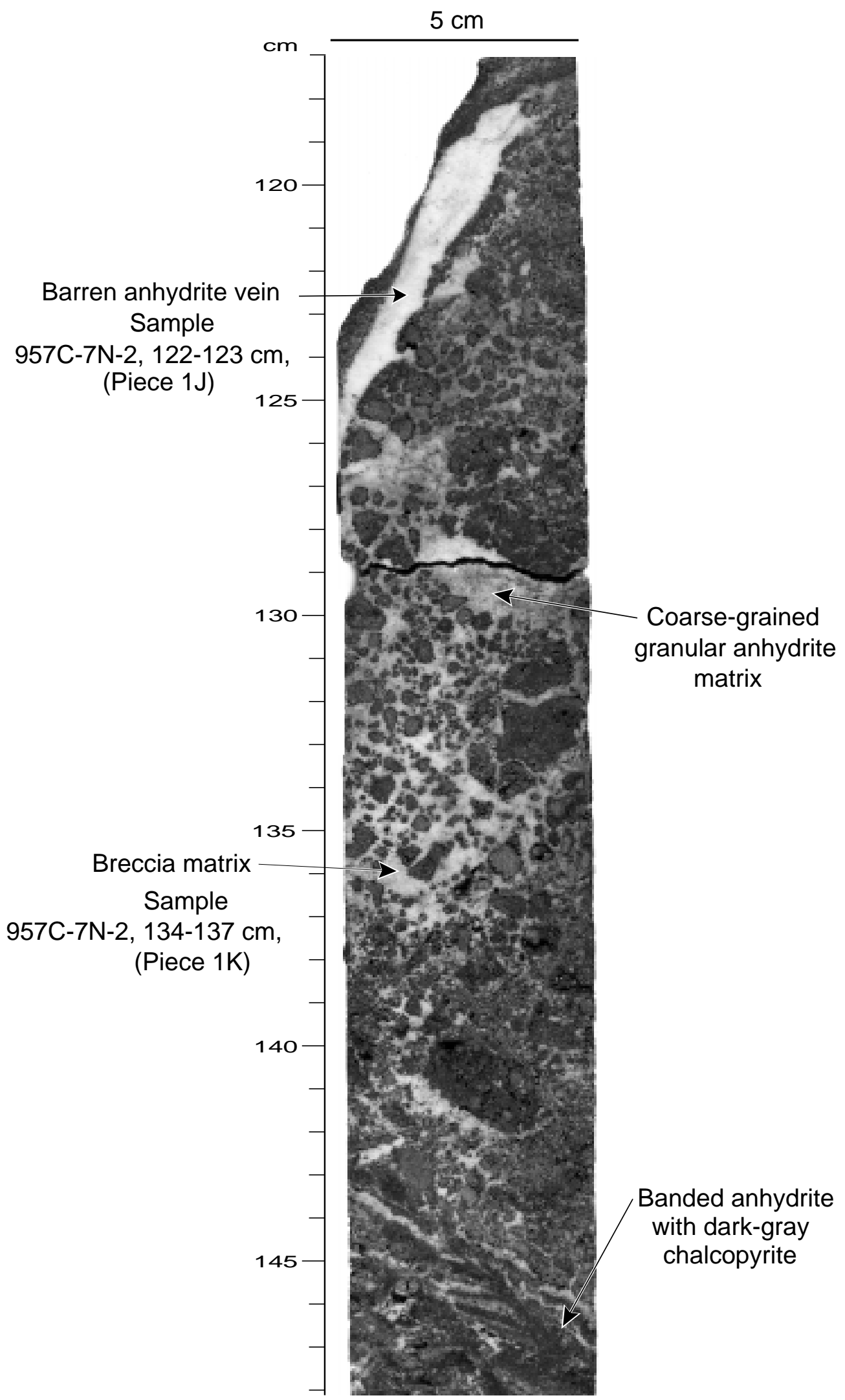

Figure 3. Nodular pyrite-anhydrite breccia (Sample 158-957C-7N-2, 120-150 cm [Pieces 1J and 1K]) showing anhydrite as breccia matrix, as vein filling with disseminated chalcopyrite, and as a later barren anhydrite vein. Locations of samples taken are also shown. 


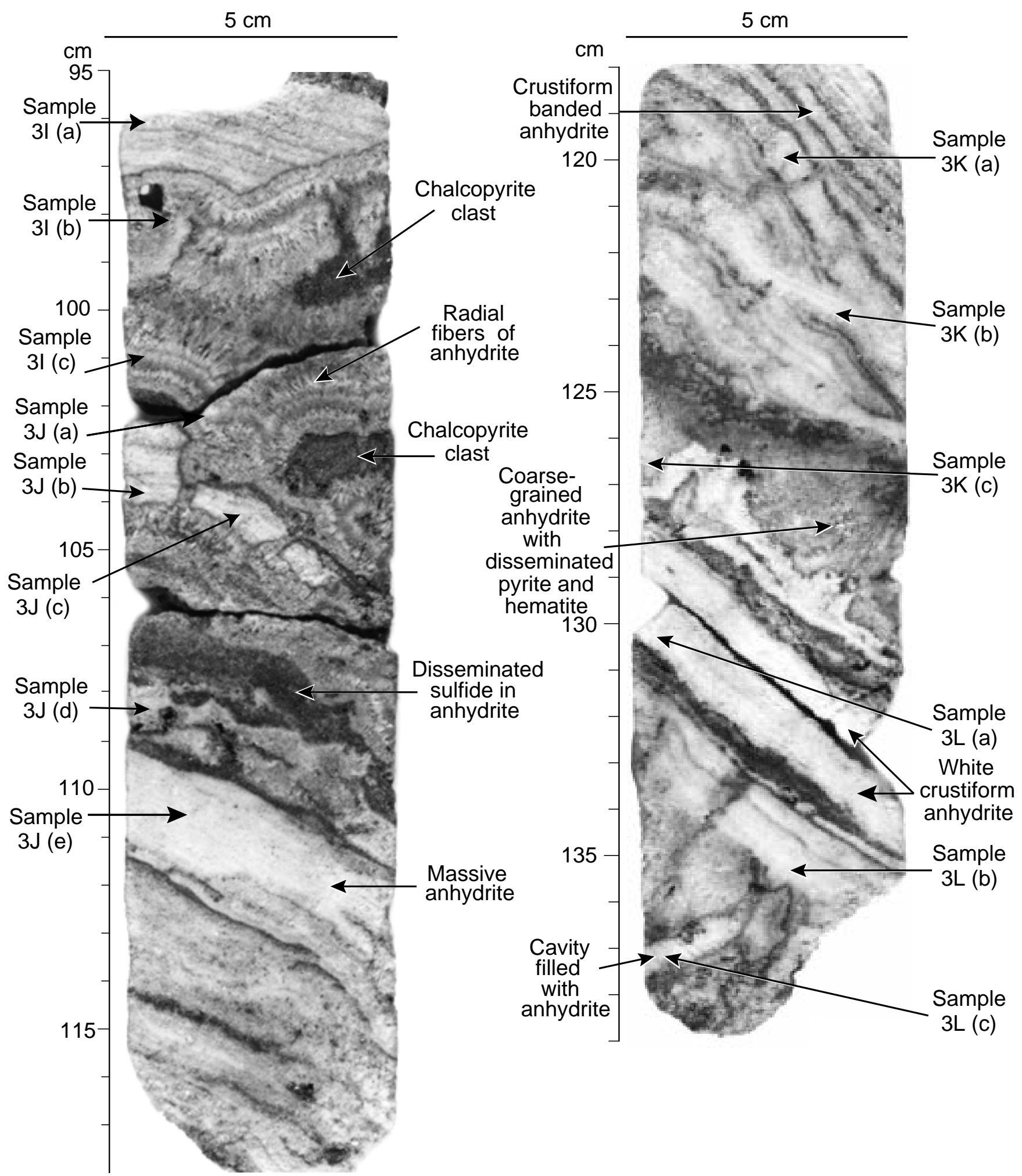

Figure 4. Multiple generations of crustiform-banded anhydrite with clasts of chalcopyrite (Sample 158-957C-11N-1, 95-139 cm [Pieces 3I, J, K, L]). Note the coarse anhydrite crystal growth around clasts and in the center of late-stage bands, and minor disseminated sulfides and hematite. Sample locations are identified by the piece number followed by a letter. 


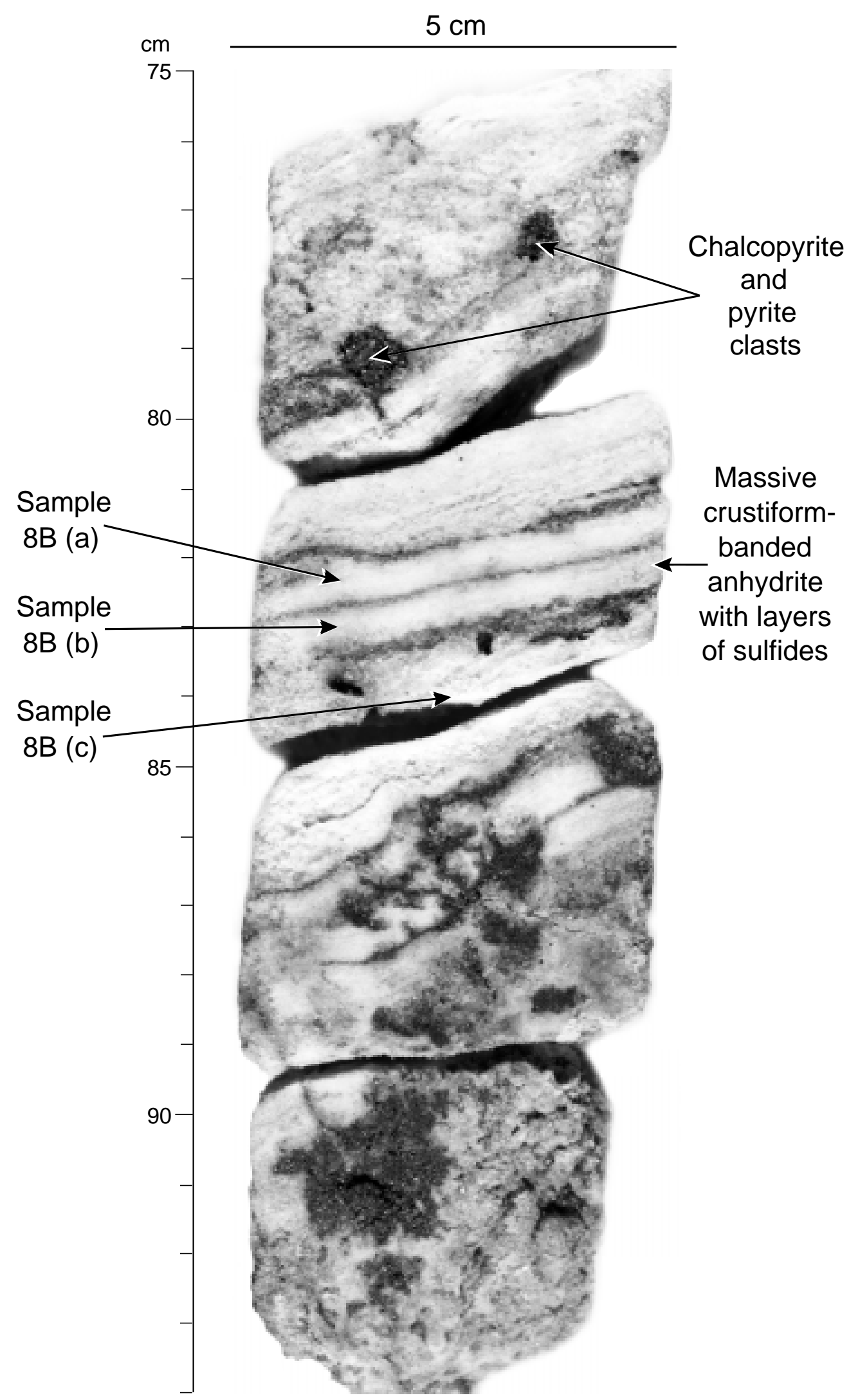

Figure 5. Massive crustiform-banded anhydrite vein with concentrations of chalcopyrite and pyrite (Sample 158-957C-12N-1, 75-94 cm [Pieces 8A, B, C, D]). Samples were taken from adjacent growth bands in Piece 8B. 
Table 1. Anhydrite samples selected for REE analyses.

\begin{tabular}{|c|c|c|c|c|}
\hline $\begin{array}{l}\text { Hole, core, } \\
\text { section, interval }(\mathrm{cm})\end{array}$ & Piece & $\begin{array}{l}\text { Depth of } \\
\text { piece } \\
\text { (mbsf) }\end{array}$ & Anhydrite occurrence & Rock type \\
\hline \multicolumn{5}{|l|}{ TAG-1 AREA } \\
\hline $957 \mathrm{C}-6 \mathrm{~W}-1,0-2$ & 1 & 15.00 & Breccia matrix & Pyrite-anhydrite breccia \\
\hline $957 \mathrm{C}-7 \mathrm{~N}-1,73-74$ & $8 \mathrm{~A}$ & 20.22 & Anhydrite from broken surface of piece, likely a vein & Nodular siliceous pyrite-anhydrite breccia \\
\hline $957 \mathrm{C}-7 \mathrm{~N}-1,83-86$ & 8B (a) & 20.33 & Breccia matrix & Nodular siliceous pyrite-anhydrite breccia \\
\hline $957 \mathrm{C}-7 \mathrm{~N}-1,91-94$ & $8 \mathrm{~B}(\mathrm{~b})$ & 20.33 & Crustiform banded vein with thin pyrite-rich bands & Nodular siliceous pyrite-anhydrite breccia \\
\hline $957 \mathrm{C}-7 \mathrm{~N}-1,98-99$ & $8 \mathrm{~B}(\mathrm{c})$ & 20.33 & Barren vein & Nodular siliceous pyrite-anhydrite breccia \\
\hline $957 \mathrm{G}-3 \mathrm{~N}-1,72-74$ & 6 & 21.68 & Coarse-grained vein & Nodular siliceous pyrite-anhydrite breccia \\
\hline $957 \mathrm{C}-7 \mathrm{~N}-2,122-123$ & $1 \mathrm{~J}$ & 22.03 & Barren vein (see Fig. 3) & Nodular siliceous pyrite-anhydrite breccia \\
\hline $957 \mathrm{C}-7 \mathrm{~N}-2,134-137$ & $1 \mathrm{~K}$ & 22.12 & Breccia matrix (see Fig. 3) & Nodular siliceous pyrite-anhydrite breccia \\
\hline $957 \mathrm{C}-11 \mathrm{~N}-1,96-97$ & 3I (a) & 31.65 & $\begin{array}{l}\text { Finely }(2-4 \mathrm{~mm} \text { ) banded portion of complex crustiform-banded vein (see } \\
\text { Fig. 4) }\end{array}$ & Anhydrite vein in pyrite-silica-anhydrite breccia \\
\hline $957 \mathrm{C}-11 \mathrm{~N}-1,97-98$ & $3 \mathrm{I}(\mathrm{b})$ & 31.65 & $\begin{array}{l}\text { Radial fibers around vug in band of complex crustiform-banded vein (see } \\
\text { Fig. 4) }\end{array}$ & Anhydrite vein in pyrite-silica-anhydrite breccia \\
\hline $957 \mathrm{C}-11 \mathrm{~N}-1,100-101$ & $3 \mathrm{I}(\mathrm{c})$ & 31.65 & Radial fibers from band of complex crustiform-banded vein (see Fig. 4) & Anhydrite vein in pyrite-silica-anhydrite breccia \\
\hline $957 \mathrm{C}-11 \mathrm{~N}-1,102-103$ & $3 \mathrm{~J}(\mathrm{a})$ & 31.71 & $\begin{array}{l}\text { Radial fibers from broken surface of complex crustiform-banded vein (see } \\
\text { Fig. 4) }\end{array}$ & Anhydrite vein in pyrite-silica-anhydrite breccia \\
\hline $957 \mathrm{C}-11 \mathrm{~N}-1,103-104$ & $3 \mathrm{~J}(\mathrm{~b})$ & 31.71 & Cavity filling within complex crustiform-banded vein (see Fig. 4) & Anhydrite vein in pyrite-silica-anhydrite breccia \\
\hline $957 \mathrm{C}-11 \mathrm{~N}-1,104-105$ & $3 \mathrm{~J}(\mathrm{c})$ & 31.71 & Cavity filling within complex crustiform-banded vein (see Fig. 4) & Anhydrite vein in pyrite-silica-anhydrite breccia \\
\hline $957 \mathrm{C}-11 \mathrm{~N}-1,108-109$ & $3 \mathrm{~J}(\mathrm{~d})$ & 31.71 & Massive granular vein with abundant disseminated sulfides (see Fig. 4) & Anhydrite vein in pyrite-silica-anhydrite breccia \\
\hline $957 \mathrm{C}-11 \mathrm{~N}-1,111-112$ & $3 \mathrm{~J}(\mathrm{e})$ & 31.71 & Massive granular vein with less abundant disseminated sulfides (see Fig. 4) & Anhydrite vein in pyrite-silica-anhydrite breccia \\
\hline $957 \mathrm{C}-11 \mathrm{~N}-1,120-121$ & $3 \mathrm{~K}(\mathrm{a})$ & 31.88 & Growth band within crustiform banded vein (see Fig. 4) & Anhydrite vein in pyrite-silica-anhydrite breccia \\
\hline $957 \mathrm{C}-11 \mathrm{~N}-1,123-124$ & $3 \mathrm{~K}(\mathrm{~b})$ & 31.88 & Growth band within same crustiform banded vein as (a) (see Fig. 4) & Anhydrite vein in pyrite-silica-anhydrite breccia \\
\hline $957 \mathrm{C}-11 \mathrm{~N}-1,125-126$ & $3 \mathrm{~K}(\mathrm{c})$ & 31.88 & Growth band within crustiform same banded vein as (a) (see Fig. 4) & Anhydrite vein in pyrite-silica-anhydrite breccia \\
\hline $957 \mathrm{C}-11 \mathrm{~N}-1,130-131$ & $3 \mathrm{~L}$ (a) & 32.00 & White crustiform anhydrite in complex crustiform-banded vein (see Fig. 4) & Anhydrite vein in pyrite-silica-anhydrite breccia \\
\hline $957 \mathrm{C}-11 \mathrm{~N}-1,134-135$ & $3 \mathrm{~L}(\mathrm{~b})$ & 32.00 & Same as (a) but from other side of band of disseminated sulfides (see Fig. 4) & Anhydrite vein in pyrite-silica-anhydrite breccia \\
\hline $957 \mathrm{C}-11 \mathrm{~N}-1,137-138$ & $3 \mathrm{~L}(\mathrm{c})$ & 32.00 & Cavity filling within complex crustiform-banded vein (see Fig. 4) & Anhydrite vein in pyrite-silica-anhydrite breccia \\
\hline $957 \mathrm{C}-11 \mathrm{~N}-2,57-59$ & $1 \mathrm{H}$ & 32.60 & Massive granular anhydrite vein with chalcopyrite selvage & Pyrite-silica-anhydrite breccia \\
\hline $957 \mathrm{C}-11 \mathrm{~N}-2,63-64$ & $1 \mathrm{~J}$ & 32.73 & Massive granular anhydrite vein with chalcopyrite selvage & Pyrite-silica-anhydrite breccia \\
\hline $957 \mathrm{C}-11 \mathrm{~N}-2,70-72$ & $1 \mathrm{~K}$ & 32.80 & Breccia matrix & Pyrite-silica-anhydrite breccia \\
\hline $957 \mathrm{C}-11 \mathrm{~N}-3,66-68$ & 6 & 34.16 & Center of vein & Anhydrite vein in pyrite-silica-anhydrite breccia \\
\hline $957 \mathrm{C}-12 \mathrm{~N}-1,82-83$ & $8 \mathrm{~B}(\mathrm{a})$ & 35.58 & Growth band within crustiform-banded vein (see Fig. 5) & Anhydrite vein in pyrite-silica-anhydrite breccia \\
\hline $957 \mathrm{C}-12 \mathrm{~N}-1,82-83$ & $8 \mathrm{~B}(\mathrm{~b})$ & 35.58 & Growth band within crustiform-banded vein (see Fig. 5) & Anhydrite vein in pyrite-silica-anhydrite breccia \\
\hline $957 \mathrm{C}-12 \mathrm{~N}-1,83-84$ & $8 \mathrm{~B}(\mathrm{c})$ & 35.58 & Growth band within crustiform-banded vein (see Fig. 5) & Anhydrite vein in pyrite-silica-anhydrite breccia \\
\hline $957 \mathrm{C}-14 \mathrm{~N}-2,56-60$ & 3 & 41.45 & Crustiform-banded anhydrite vein & Anhydrite vein in pyrite-silica breccia \\
\hline $957 \mathrm{C}-15 \mathrm{~N}-2,30-31$ & $1 \mathrm{~F}$ & 43.39 & Narrow vein up to $5 \mathrm{~mm}$ wide & Anhydrite vein in pyrite-silica breccia \\
\hline $957 \mathrm{E}-5 \mathrm{R}-1,9-10$ & 3 & 58.68 & Anhydrite from broken surface; narrow vein? & Pyrite-silica breccia \\
\hline \multicolumn{5}{|l|}{ TAG-2 AREA } \\
\hline $957 \mathrm{H}-1 \mathrm{~N}-1,38-45$ & 9 & 9.07 & Vein, $1-5 \mathrm{~mm}$ wide & Porous massive pyrite \\
\hline $957 \mathrm{H}-5 \mathrm{~N}-1,70-72$ & 7 & 27.35 & Vein, up to $1 \mathrm{~cm}$ wide & Nodular pyrite-silica breccia \\
\hline $957 \mathrm{H}-5 \mathrm{~N}-1,76-77$ & 7 & 27.35 & Crystals from broken surface; likely vein material & Nodular pyrite-silica breccia \\
\hline $957 \mathrm{H}-5 \mathrm{~N}-2,8-10$ & 1B & 27.68 & Coarse-grained crystals from several vugs & Silicified wallrock breccia \\
\hline $957 \mathrm{H}-5 \mathrm{~N}-2,55-57$ & 2 & 28.07 & Coarse-grained crystals from vein & Silicified wallrock breccia \\
\hline $957 \mathrm{H}-5 \mathrm{~N}-2,55-57$ & 2 & 28.07 & Coarse-grained crystals from vein along broken surface & Silicified wallrock breccia \\
\hline \multicolumn{5}{|l|}{ TAG-5 AREA } \\
\hline $957 \mathrm{O}-4 \mathrm{R}-1,2-4$ & 1 & 15.90 & Broken surface-likely a small vein & Vein-related pyrite-anhydrite breccia \\
\hline
\end{tabular}

Notes: The inclusion of "anhydrite vein" in the "Rock type" description implies that the entire piece was part of the anhydrite vein, and that the host rock type has been determined by the rock types above and below. When only a rock type is given, the anhydrite-bearing structure was only a small component within the host rock.

Eu* $_{\mathrm{N}}$ value of 13.7. Although the absolute REE concentrations are higher, these chondrite-normalized patterns are similar to that for end-member black smoker hydrothermal fluid (Mitra et al., 1994), which shows an $\mathrm{Nd}_{\mathrm{N}} / \mathrm{Yb}_{\mathrm{N}}$ of 8.8 and an $\mathrm{Eu}_{\mathrm{N}} / \mathrm{Eu}^{*}{ }_{\mathrm{N}}$ of 9.1 . However, they are distinctly different from the REE pattern for anhydrite from a black smoker chimney reported by Mills and Elderfield (1995) and shown in Figure 8, which is similarly enriched in LREEs $\left(\mathrm{Nd}_{\mathrm{N}} / \mathrm{Yb}_{\mathrm{N}}\right.$ of 13.9), but shows a slight negative Eu anomaly $\left(\mathrm{Eu}_{\mathrm{N}} / \mathrm{Eu}^{*}{ }_{\mathrm{N}}\right.$ of 0.9$)$. No systematic variations with depth in the absolute REE concentrations, degree of LREE enrichment, or magnitude of the Eu anomaly are evident in the anhydrite from the TAG-1 area.

The chondrite-normalized REE patterns of anhydrites from the TAG-2 area (Fig. 9) are similar to those from the TAG-1 area, and show LREE enrichment with $\mathrm{Nd}_{\mathrm{N}} / \mathrm{Yb}_{\mathrm{N}}$ values ranging from 8.5 to 12.6. However, they tend to exhibit $\mathrm{Eu}_{\mathrm{N}} / \mathrm{Eu}^{*}{ }_{\mathrm{N}}$ values $(6.3-18.5)$ at the upper end and extending beyond the range shown by the TAG-1 samples. One sample of anhydrite-Sample 957H-5N-2, 8-10 cm (Piece 1B) - contains the highest absolute REE concentrations of any sample from TAG-1 or TAG-2. It shows a similar degree of LREE enrichment $\left(\mathrm{Nd}_{\mathrm{N}} / \mathrm{Yb}_{\mathrm{N}}=9.6\right)$ but a Eu anomaly $\left(\mathrm{Eu}_{\mathrm{N}} / \mathrm{Eu}^{*}{ }_{\mathrm{N}}=6.4\right)$ that is smaller than other samples from the TAG-2 area, but is similar to the TAG-1 anhydrites. The chondrite-normalized REE pattern of an anhydrite sample from a white smoker chimney from the same area (Fig. 8) is similar, although it shows more LREE enrichment $\left(\mathrm{Nd}_{\mathrm{N}} /\right.$ $\left.\mathrm{Yb}_{\mathrm{N}}=22.2\right)$ and a larger Eu anomaly $\left(\mathrm{Eu}_{\mathrm{N}} / \mathrm{Eu}^{*}{ }_{\mathrm{N}}=20.2\right)$. Comparison of the REE patterns in these samples with that of the white smoker hydrothermal fluid (Mitra et al., 1994) indicates that the fluid shows a similar degree of LREE enrichment $\left(\mathrm{Nd}_{\mathrm{N}} / \mathrm{Yb}_{\mathrm{N}}=11.6\right)$ but a considerably greater Eu anomaly $\left(\mathrm{Eu}_{\mathrm{N}} / \mathrm{Eu}^{*}{ }_{\mathrm{N}}=126.9\right)$. As in the TAG-1 area, there do not appear to be any systematic changes in the REE concentrations or chondrite-normalized patterns with depth.

The chondrite-normalized pattern of the one anhydrite sample from Hole 9570 in the TAG-5 area (Fig. 9) shows some striking differences from all other samples analyzed. The absolute REE concentrations in this sample, with the exception of $\mathrm{Eu}$, are the highest found in this study. Although the $\mathrm{Nd}_{N} / \mathrm{Yb}_{\mathrm{N}}$ value of 11.7 is not significantly different, La appears to be depleted relative to the other LREEs. In addition, the Eu anomaly, although still positive, is extremely small $\left(\mathrm{Eu}_{\mathrm{N}} / \mathrm{Eu}^{*}{ }_{\mathrm{N}}\right.$ of 1.5$)$. The only sample with a REE pattern to which this one shows any similarity is an anhydrite sample from a black smoker chimney (Mills and Elderfield, 1995), which is shown in Figure 8. However, although this has a similar degree of LREE enrichment $\left(\mathrm{Nd}_{\mathrm{N}} / \mathrm{Yb}_{\mathrm{N}}=13.9\right)$, it does not exhibit the depletion of La relative to $\mathrm{Ce}$ and it exhibits a slight negative Eu anomaly $\left(\mathrm{Eu}_{\mathrm{N}} / \mathrm{Eu}^{*}{ }_{\mathrm{N}}\right.$ of 0.9$)$.

\section{REE Variations in Anhydrite from Different Textural Settings}

Given the wide range in the $\mathrm{Nd}_{N} / \mathrm{Yb}_{\mathrm{N}}$ and $\mathrm{Eu}_{\mathrm{N}} / \mathrm{Eu}^{*}{ }_{\mathrm{N}}$ values observed in the sample suite, it is of interest to determine whether these variations are systematic and can be related to the textural setting of the anhydrite. 
Table 2. REE concentrations in anhydrites from the active TAG hydrothermal mound.

\begin{tabular}{|c|c|c|c|c|c|c|c|c|c|c|c|c|c|c|c|c|c|}
\hline $\begin{array}{l}\text { Hole, core, section } \\
\text { interval }(\mathrm{cm})\end{array}$ & Piece & $\begin{array}{l}\text { Depth of } \\
\text { piece } \\
\text { (mbsf) }\end{array}$ & Group & $\begin{array}{c}\mathrm{La} \\
(\mathrm{ppm})\end{array}$ & $\begin{array}{c}\mathrm{Ce} \\
(\mathrm{ppm})\end{array}$ & $\begin{array}{c}\mathrm{Pr} \\
(\mathrm{ppm})\end{array}$ & $\begin{array}{c}\mathrm{Nd} \\
(\mathrm{ppm})\end{array}$ & $\begin{array}{c}\mathrm{Sm} \\
(\mathrm{ppm})\end{array}$ & $\begin{array}{c}\mathrm{Eu} \\
(\mathrm{ppm})\end{array}$ & $\begin{array}{c}\mathrm{Gd} \\
(\mathrm{ppm})\end{array}$ & $\begin{array}{c}\mathrm{Tb} \\
(\mathrm{ppm})\end{array}$ & $\begin{array}{c}\text { Dy } \\
(\mathrm{ppm})\end{array}$ & $\begin{array}{c}\mathrm{Ho} \\
(\mathrm{ppm})\end{array}$ & $\begin{array}{c}\mathrm{Er} \\
(\mathrm{ppm})\end{array}$ & $\begin{array}{l}\mathrm{Tm} \\
(\mathrm{ppm})\end{array}$ & $\begin{array}{c}\mathrm{Yb} \\
(\mathrm{ppm})\end{array}$ & $\begin{array}{c}\mathrm{Lu} \\
(\mathrm{ppm})\end{array}$ \\
\hline \multicolumn{18}{|l|}{ TAG-1 AREA } \\
\hline $957 \mathrm{C}-6 \mathrm{~W}-1,0-2$ & 1 & 15.00 & I & 0.527 & 1.051 & 0.144 & 0.610 & 0.147 & 0.398 & 0.104 & 0.019 & 0.094 & 0.016 & 0.032 & 0.005 & 0.031 & 0.005 \\
\hline $957 \mathrm{C}-7 \mathrm{~N}-1,73-74$ & $8 \mathrm{~A}$ & 20.22 & II & 0.532 & 1.046 & 0.145 & 0.607 & 0.156 & 0.318 & 0.149 & 0.023 & 0.107 & 0.017 & 0.032 & 0.005 & 0.031 & 0.005 \\
\hline $957 \mathrm{C}-7 \mathrm{~N}-1,83-86$ & $8 \mathrm{~B}(\mathrm{a})$ & 20.33 & I & 0.453 & $\begin{array}{l}1.054 \\
1.050\end{array}$ & 0.160 & 0.726 & 0.179 & 0.449 & 0.137 & 0.021 & 0.096 & 0.016 & 0.032 & 0.005 & 0.025 & 0.003 \\
\hline $957 \mathrm{C}-7 \mathrm{~N}-1,91-94$ & $8 \mathrm{~B}$ (b) & 20.33 & II & 0.501 & 1.205 & 0.189 & 0.897 & 0.234 & 0.486 & 0.181 & 0.026 & 0.128 & 0.022 & 0.051 & 0.006 & 0.031 & 0.005 \\
\hline $957 \mathrm{C}-7 \mathrm{~N}-1,98-99$ & $8 \mathrm{~B}(\mathrm{c})$ & 20.33 & I & 0.484 & 1.014 & 0.150 & 0.627 & 0.147 & 0.589 & 0.107 & 0.020 & 0.085 & 0.012 & 0.023 & 0.003 & $\begin{array}{l}0.019 \\
0.019\end{array}$ & 0.003 \\
\hline $957 \mathrm{G}-3 \mathrm{~N}-1,72-74$ & 6 & 21.68 & I & 0.360 & 0.922 & 0.139 & 0.643 & 0.167 & 0.425 & 0.118 & 0.015 & 0.089 & 0.012 & 0.023 & 0.003 & 0.013 & 0.001 \\
\hline $957 \mathrm{C}-7 \mathrm{~N}-2,122-123$ & $1 \mathrm{~J}$ & 22.03 & II & 0.606 & 1.423 & 0.211 & 0.960 & 0.242 & 0.450 & 0.182 & 0.024 & 0.125 & 0.020 & 0.035 & 0.005 & 0.027 & 0.003 \\
\hline $957 \mathrm{C}-7 \mathrm{~N}-2,134-137$ & $1 \mathrm{~K}$ & 22.12 & II & 0.493 & 1.116 & 0.167 & 0.783 & 0.208 & 0.360 & 0.160 & 0.024 & 0.124 & 0.019 & 0.043 & 0.007 & 0.034 & 0.006 \\
\hline $957 \mathrm{C}-11 \mathrm{~N}-1,96-97$ & $3 \mathrm{I}$ (a) & 31.65 & I & 0.652 & 1.380 & 0.186 & 0.770 & 0.168 & 0.473 & 0.115 & 0.017 & 0.093 & 0.013 & 0.025 & 0.004 & 0.020 & 0.005 \\
\hline $957 \mathrm{C}-11 \mathrm{~N}-1,97-98$ & $3 \mathrm{I}(\mathrm{b})$ & 31.65 & I & 0.559 & 1.622 & 0.272 & 1.381 & 0.386 & 0.824 & 0.293 & 0.037 & 0.185 & 0.025 & 0.042 & 0.006 & 0.020 & 0.003 \\
\hline $957 \mathrm{C}-11 \mathrm{~N}-1,100-101$ & $3 \mathrm{I}(\mathrm{c})$ & 31.65 & I & 0.552 & 1.500 & 0.238 & 1.182 & 0.352 & 0.741 & 0.249 & 0.033 & 0.155 & 0.022 & 0.039 & 0.006 & 0.017 & 0.002 \\
\hline $957 \mathrm{C}-11 \mathrm{~N}-1,102-103$ & $3 \mathrm{~J}$ (a) & 31.71 & I & 0.653 & 1.747 & 0.288 & 1.401 & 0.429 & 0.863 & 0.280 & 0.044 & 0.201 & 0.025 & 0.055 & 0.007 & 0.033 & 0.005 \\
\hline $957 \mathrm{C}-11 \mathrm{~N}-1,103-104$ & $3 \mathrm{~J}(\mathrm{~b})$ & 31.71 & I & 0.577 & 1.345 & 0.193 & 0.945 & 0.238 & 0.612 & 0.175 & 0.025 & 0.126 & 0.020 & 0.040 & 0.006 & 0.028 & 0.006 \\
\hline $957 \mathrm{C}-11 \mathrm{~N}-1,104-105$ & $3 \mathrm{~J}(\mathrm{c})$ & 31.71 & I & 0.601 & 1.391 & 0.205 & 0.945 & 0.241 & 0.612 & 0.164 & 0.027 & 0.124 & 0.018 & 0.041 & 0.006 & 0.032 & 0.004 \\
\hline $957 \mathrm{C}-11 \mathrm{~N}-1,108-109$ & $3 \mathrm{~J}(\mathrm{~d})$ & 31.71 & I & 0.853 & 2.301 & 0.363 & 1.688 & 0.428 & 0.828 & 0.303 & 0.044 & 0.229 & 0.034 & 0.081 & 0.009 & 0.042 & 0.006 \\
\hline $957 \mathrm{C}-11 \mathrm{~N}-1,111-112$ & $3 \mathrm{~J}(\mathrm{e})$ & 31.71 & I & 1.105 & 2.646 & 0.387 & $\begin{array}{l}1.000 \\
1.711\end{array}$ & 0.423 & $\begin{array}{l}0.020 \\
0.940\end{array}$ & 0.285 & 0.047 & 0.226 & 0.035 & $\begin{array}{l}0.001 \\
0.079\end{array}$ & 0.011 & 0.054 & 0.007 \\
\hline $957 \mathrm{C}-11 \mathrm{~N}-1,12$ & $3 \mathrm{~K}$ (a) & 31.88 & II & 0.654 & 1.517 & 0.234 & 1.105 & 0.296 & 0.302 & 0.227 & 0.038 & 0.187 & 0.030 & 0.066 & 10 & 48 & 0.007 \\
\hline $957 \mathrm{C}-11$ & $3 \mathrm{~K}$ (b) & 31.88 & II & 0.479 & 1.140 & 0.193 & 0.971 & 0.280 & 0.220 & 0.248 & 0.040 & 0.183 & 0.032 & 0.071 & 0.009 & 0.045 & 0.006 \\
\hline $957 \mathrm{C}-11 \mathrm{~N}-1,125-126$ & $3 \mathrm{~K}(\mathrm{c})$ & 31.88 & II & 0.753 & 1.852 & 0.303 & 1.651 & 0.504 & 0.349 & 0.462 & 0.070 & 0.352 & 0.059 & 0.146 & 0.018 & 0.116 & 0.015 \\
\hline $957 \mathrm{C}-11 \mathrm{~N}-1,130-131$ & $3 \mathrm{~L}$ (a) & 32.00 & II & 0.613 & 1.481 & 0.224 & $\begin{array}{l}1.031 \\
1.060\end{array}$ & 0.269 & 0.400 & 0.209 & 0.028 & 0.157 & 0.024 & 0.047 & 0.007 & 0.035 & 0.005 \\
\hline $957 \mathrm{C}-11 \mathrm{~N}-1,134-135$ & $3 \mathrm{~L}$ (b) & 32.00 & II & 0.666 & 1.554 & 0.229 & 1.046 & 0.239 & 0.376 & 0.186 & 0.027 & 0.150 & 0.021 & 0.051 & 0.009 & 0.040 & 0.006 \\
\hline $957 \mathrm{C}-11 \mathrm{~N}-1,137-138$ & $3 \mathrm{~L}(\mathrm{c})$ & 32.00 & II & 0.500 & 1.194 & 0.173 & 0.815 & 0.207 & 0.364 & 0.184 & 0.026 & 0.137 & 0.023 & 0.047 & 0.007 & 0.037 & 0.006 \\
\hline $957 \mathrm{C}-11 \mathrm{~N}-2,57-59$ & $1 \mathrm{H}$ & 32.60 & II & 0.538 & 1.338 & 0.209 & 0.993 & 0.269 & 0.312 & 0.259 & 0.035 & 0.176 & 0.027 & 0.062 & 0.008 & 0.040 & 0.005 \\
\hline $957 \mathrm{C}-11 \mathrm{~N}-2,63-64$ & $1 \mathrm{~J}$ & 32.73 & I & 0.627 & 1.490 & 0.226 & 1.040 & 0.263 & 0.585 & 0.193 & 0.028 & 0.151 & 0.022 & 0.040 & 0.005 & 0.023 & 0.005 \\
\hline $957 \mathrm{C}-11 \mathrm{~N}-2,70-72$ & $1 \mathrm{~K}$ & 32.80 & I & 0.661 & 1.359 & 0.193 & 0.793 & 0.196 & 0.545 & 0.131 & 0.022 & 0.103 & 0.015 & 0.038 & 0.005 & 0.033 & 0.005 \\
\hline $957 \mathrm{C}-11 \mathrm{~N}-3,66-68$ & 6 & 34.16 & II & 0.656 & 1.778 & 0.291 & 1.531 & 0.429 & 0.359 & 0.352 & 0.053 & 0.275 & 0.041 & 0.088 & 0.011 & 0.051 & 0.006 \\
\hline $957 \mathrm{C}-12 \mathrm{~N}-1,82-83$ & $8 \mathrm{~B}(\mathrm{a})$ & 35.58 & II & 0.728 & $\begin{array}{l}1.858 \\
1.858\end{array}$ & 0.297 & 1.475 & 0.422 & 0.333 & 0.358 & 0.055 & 0.259 & 0.043 & $\begin{array}{l}0.000 \\
0.100\end{array}$ & 0.013 & 0.068 & 0.008 \\
\hline $957 \mathrm{C}-12 \mathrm{~N}-1,82-83$ & $8 \mathrm{~B}(\mathrm{~b})$ & 35.58 & II & 0.666 & $\begin{array}{l}1.030 \\
1.710\end{array}$ & 0.277 & 1.408 & 0.374 & 0.318 & 0.327 & 0.0 & 0.265 & 0.043 & 0.101 & 0.013 & 0.071 & $\begin{array}{l}0.009 \\
0.009\end{array}$ \\
\hline$-1,83-84$ & $8 \mathrm{~B}(\mathrm{c})$ & 35 . & II & 0.5 & 1.4 & 0.243 & $\begin{array}{l}1.400 \\
1.288\end{array}$ & 0.3 & 0. & 0. & 0 & 0 & 0. & 0.084 & 0 & 0 & .008 \\
\hline $957 \mathrm{C}-14$ & 3 & 41. & I & 0.925 & 2.031 & 0.283 & $\begin{array}{l}1.270 \\
1.277\end{array}$ & 0. & 0. & 0. & 0. & 0.1 & 0.029 & 0.063 & 0.008 & 0.052 & 0.008 \\
\hline $957 \mathrm{C}-15 \mathrm{~N}-2,30-31$ & $1 \mathrm{~F}$ & 43. & II & 0.579 & 1.344 & 0.205 & 0.986 & 0.279 & 0.497 & 0.207 & 0.029 & 0.1 & 0.029 & 0.062 & 0.008 & 0 & 0.006 \\
\hline $957 \mathrm{E}-5 \mathrm{R}-1,9-10$ & 3 & 58.68 & II & 0.685 & 1.795 & 0.273 & 1.296 & 0.322 & 0.620 & 0.251 & 0.037 & 0.184 & 0.029 & 0.071 & 0.008 & 0.046 & 0.005 \\
\hline \multicolumn{18}{|l|}{ TAG-2 AREA } \\
\hline $957 \mathrm{H}-1 \mathrm{~N}-1,38-45)$ & 9 & 9.07 & & 0.773 & 1.43 & 0.169 & 0.615 & 0.127 & 0.734 & 0.1 & 0.0 & 0.0 & 0.012 & 0.032 & 0.004 & 0.026 & 0.003 \\
\hline $957 \mathrm{H}-5 \mathrm{~N}-1,70-72$ & 7 & 27.35 & & 0.679 & 1.566 & 0.225 & 1.067 & 0.346 & 1.133 & 0.266 & 0.043 & 0.225 & 0.030 & 0.050 & 0.006 & 0.031 & 0.003 \\
\hline $957 \mathrm{H}-5 \mathrm{~N}-1,76-77$ & 7 & 27.35 & & 0.769 & 1.843 & 0.277 & 1.356 & 0.472 & 1.226 & 0.382 & 0.059 & 0.265 & 0.038 & 0.072 & 0.008 & 0.041 & 0.003 \\
\hline $957 \mathrm{H}-5 \mathrm{~N}-2,8-10$ & $1 \mathrm{~B}$ & 27.68 & & $\begin{array}{l}0.109 \\
1.500\end{array}$ & $\begin{array}{l}1.043 \\
4.121\end{array}$ & 0.624 & $\begin{array}{l}1.300 \\
3.129\end{array}$ & $\begin{array}{l}.412 \\
1.060\end{array}$ & $\begin{array}{l}1.220 \\
1.990\end{array}$ & 0.785 & 0.132 & 0.613 & 0.088 & 0.187 & 0.023 & 0.119 & 0.012 \\
\hline $957 \mathrm{H}-5 \mathrm{~N}-2,55-57$ & 2 & 28.07 & & 0.402 & 0.869 & 0.135 & 0.618 & 0.2 & 0.825 & 0.185 & 0.027 & 0.158 & 0.024 & 0.039 & 0.005 & 0.030 & 0.004 \\
\hline $957 \mathrm{H}-5 \mathrm{~N}-2,55-57$ & 2 & 28.07 & & 0.641 & 1.318 & 0.199 & 0.945 & 0.333 & 0.881 & 0.262 & 0.044 & 0.198 & 0.029 & 0.055 & 0.006 & 0.032 & 0.003 \\
\hline \multicolumn{18}{|l|}{ TAG-5 AREA } \\
\hline $957 \mathrm{O}-4 \mathrm{R}-1,2-4 \mathrm{~cm}$ & 1 & 15.90 & & 1.720 & 6.033 & 1.108 & 6.145 & 1.852 & 0.860 & 1.511 & 0.225 & 1.124 & 0.175 & 0.385 & 0.041 & 0.191 & 0.020 \\
\hline \multicolumn{18}{|l|}{ FLUIDS } \\
\hline Black smoker fluid ( $\mathrm{p}$ & & & & 5.68 & 13.20 & - & 8.68 & 1.87 & 5.42 & 1.70 & - & 1.23 & - & 0.493 & - & 0.360 & 0.044 \\
\hline White smoker fluid (p & & & & 3.40 & 4.79 & 二 & $\begin{array}{l}0.00 \\
2.14\end{array}$ & 0.358 & 13.20 & 0.256 & 二 & 0.179 & 二 & 0.0796 & 二 & 0.067 & 0.008 \\
\hline Seawater at $3400 \mathrm{~m}\left(\mathrm{ppm} \times 10^{-6}\right)$ & & & & 3.54 & 0.762 & - & 3.09 & 0.621 & 0.161 & 0.983 & - & 1.03 & - & 0.915 & - & 0.938 & 0.154 \\
\hline
\end{tabular}

Notes: Fluids data are from Mitra et al. (1994). Black smoker fluid data have been recalculated for $\mathrm{Mg}=0$; white smoker data have been recalculated for $\mathrm{Mg}=4 \mathrm{mmol} / \mathrm{kg}$. 


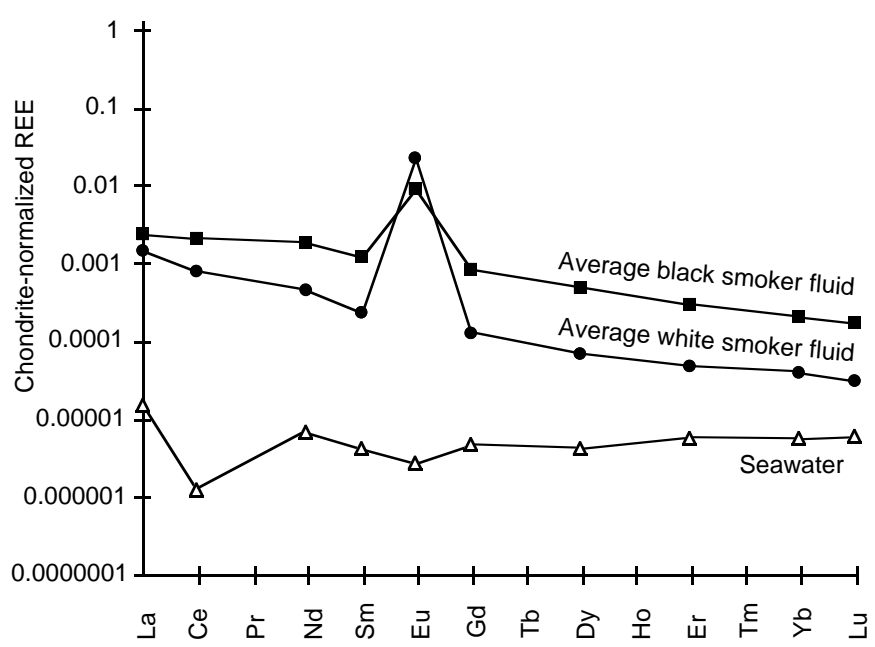

Figure 6. Chondrite-normalized REE patterns for average black and white smoker fluids and seawater from the TAG area at $3500 \mathrm{~m}$ depth. Data are from Mitra et al. (1994) and are included in Table 2.

Four samples of anhydrite from the matrix of breccias from TAG1 were analyzed: one from a pyrite-anhydrite breccia, two from siliceous pyrite-anhydrite breccias, and one from a pyrite-silica-anhydrite breccia, and their chondrite-normalized REE patterns are shown in Figure 10. They are all extremely similar, and have an average $\mathrm{Nd}_{\mathrm{N}} / \mathrm{Yb}_{\mathrm{N}}$ value of 8.73 and an average $\mathrm{Eu}_{\mathrm{N}} / \mathrm{Eu}^{*}{ }_{\mathrm{N}}$ value of 8.34. This suggests that, between 15 and $33 \mathrm{mbsf}$, precipitation of anhydrite as breccia matrix occurred under the same physical conditions and from a hydrothermal fluid of the same chemical composition.

All other samples from the TAG-1 area are from veins and, as has previously been discussed, the chondrite-normalized REE patterns are all similar and show little difference from the anhydrite in the breccia matrix. For example, Figure 11 shows the REE from the three sets of samples for which analyses of both vein and breccia matrix material are available. All three types of veins are represented among these samples, and the $\mathrm{Nd}_{\mathrm{N}} / \mathrm{Yb}_{\mathrm{N}}$ and $\mathrm{Eu}_{\mathrm{N}} / \mathrm{Eu}^{*}{ }_{\mathrm{N}}$ values vary widely as shown in the previous section; however, there do not appear to be any simple systematic variations in their REE patterns.

Analyses of anhydrite samples taken from the 45-cm-thick complex vein in Hole 957C (Fig. 4) suggest that there are variations in the REE patterns from different generations of anhydrite that can be discerned when the relationships between samples are well constrained. This is best illustrated by Figure 12, which shows a plot of $\mathrm{Nd}_{N} / \mathrm{Yb}_{\mathrm{N}}$ and $\mathrm{Eu}_{N} / \mathrm{Eu}^{*}{ }_{N}$ vs. depth. At the upper contact with the pyrite-silicaanhydrite breccia, there is a zone $\sim 2 \mathrm{~cm}$ thick of granular, banded anhydrite with sulfides disseminated along the growth bands. This is characterized by a $\mathrm{Nd}_{\mathrm{N}} / \mathrm{Yb}_{\mathrm{N}}$ value of 13.8 and a $\mathrm{Eu}_{\mathrm{N}} / \mathrm{Eu}^{*}{ }_{\mathrm{N}}$ value of 9.8. Below this zone, there is an abrupt transition into a region of coarse-grained fibers that grow in bands radiating from vein contacts and sulfide clasts. Two samples from this material show notably high $\mathrm{Nd}_{\mathrm{N}} / \mathrm{Yb}_{\mathrm{N}}$ values of $24.7-24.9$, and slightly lower $\mathrm{Eu}_{\mathrm{N}} / \mathrm{Eu}^{*}{ }_{\mathrm{N}}$ values of 7.2-7.3. Sample 957C-11N-1, 102-103 cm (Piece 3J [a]) was taken from the broken surface of Piece $3 \mathrm{~J}$ and so its textural relationship cannot be clearly established. It has a $\mathrm{Nd}_{\mathrm{N}} / \mathrm{Yb}_{\mathrm{N}}$ value of 15.3 and a $\mathrm{Eu}_{\mathrm{N}} / \mathrm{Eu}^{*}{ }_{\mathrm{N}}$ value of 8.9 , similar to Sample $957 \mathrm{C}-11 \mathrm{~N}-1,102-103 \mathrm{~cm}$ (Piece $3 \mathrm{~J}[\mathrm{~d}]$ ) with a $\mathrm{Nd}_{\mathrm{N}} / \mathrm{Yb}_{\mathrm{N}}$ value of 14.7 and $\mathrm{Eu}_{\mathrm{N}} / \mathrm{Eu}^{*}{ }_{\mathrm{N}}$ value of 8.8. Three cavities that must have formed within the fibrous anhydrite have been filled by granular anhydrite, and samples were analyzed from two of them. These samples are characterized by $\mathrm{Nd}_{N} / \mathrm{Yb}_{\mathrm{N}}$ values of 10.9-12.3, and $\mathrm{Eu}_{\mathrm{N}} / \mathrm{Eu}^{*}{ }_{\mathrm{N}}$ values of 6.7-7.1. At a depth within the section of about $110 \mathrm{~cm}$ (i.e., about $15 \mathrm{~cm}$ from the upper con- tact), there is a 2-cm-wide band of massive anhydrite with very little disseminated sulfides, and a $\mathrm{Nd}_{\mathrm{N}} / \mathrm{Yb}_{\mathrm{N}}$ value of 11.5 and a $\mathrm{Eu}_{\mathrm{N}} / \mathrm{Eu}^{*}{ }_{\mathrm{N}}$ value of 7.8 , similar to the sample from the broken surface of Piece $1 \mathrm{~J}$. The upper portion of Piece $1 \mathrm{~K}$ consists of crustiform-banded anhydrite with disseminated sulfides and some minor hematite. Three samples from different bands within this zone were taken, one of which (labeled [b]) cuts across earlier banding (labeled [a]). However, both have similar REE patterns, with lower $\mathrm{Nd}_{\mathrm{N}} / \mathrm{Yb}_{\mathrm{N}}$ values of 7.8-8.5 and $\mathrm{Eu}_{\mathrm{N}} / \mathrm{Eu}^{*}{ }_{\mathrm{N}}$ values of 2.5-3.4 than the fibrous portion above. However, one sample from a lower growth band (labeled [c]) has higher absolute concentrations of REEs, and is characterized by even lower values of $\mathrm{Nd}_{\mathrm{N}} / \mathrm{Yb}_{\mathrm{N}}$ value of 5.1 and a $\mathrm{Eu}_{\mathrm{N}} / \mathrm{Eu}^{*}{ }_{\mathrm{N}}$ value of 2.1. At a depth of about $130-133 \mathrm{~cm}$ in the section (i.e., about $35 \mathrm{~cm}$ below the upper contact), a white band of crustiform anhydrite with a distinct sulfide band occurs, and the REE patterns from this material have greater enrichment in LREEs $\left(\mathrm{Nd}_{\mathrm{N}} / \mathrm{Yb}_{\mathrm{N}}=9.5-11.1\right)$ and more distinct $\mathrm{Eu}$ anomalies $\left(\mathrm{Eu}_{\mathrm{N}} / \mathrm{Eu}^{*}{ }_{\mathrm{N}}=5.0-5.2\right)$ than the overlying anhydrite layer. Finally, the REE pattern of anhydrite filling a cavity close to this vein is similar $\left(\mathrm{Nd}_{\mathrm{N}} / \mathrm{Yb}_{\mathrm{N}}=8.0\right.$ and $\left.\mathrm{a} \mathrm{Eu}_{\mathrm{N}} / \mathrm{Eu}^{*}{ }_{\mathrm{N}}=5.6\right)$. The lower contact of this vein was not recovered intact and the upper part of the next section of core reverted to the pyrite-silica-anhydrite breccia so the nature of the contact and whether most of the vein was recovered cannot be determined.

All complex anhydrite veins that exhibit multiple growth bands do not, however, show variations in the REE concentrations or chondrite-normalized REE patterns. This is illustrated in Figure 13 in which the REE patterns are shown for three distinct growth bands within an anhydrite vein at about $35.6 \mathrm{mbsf}$. They are characterized by $\mathrm{Nd}_{\mathrm{N}} / \mathrm{Yb}_{\mathrm{N}}$ values of 7.2-9.4 and $\mathrm{Eu}_{\mathrm{N}} / \mathrm{Eu}^{*}{ }_{\mathrm{N}}$ values of 2.6-2.7.

Anhydrite samples from the TAG-2 area are mostly vein samples, except for that taken from Sample 957H-5N-2, 8-10 cm (Piece 1B), which consists of coarse-grained crystals taken from vugs. As was discussed previously, this sample has higher REE concentrations than the other samples, but a lower Eu anomaly, suggesting that this anhydrite was precipitated from a fluid that had evolved differently from that precipitating anhydrite in the veins. The other notable sample is Sample 957H-1N-1, 38-45 cm (Piece 9) from 9 mbsf, which shows a sharp decline in the LREE concentrations relative to chondrites, whereas the other samples show relatively flat LREE patterns. The sharp decline is also seen in the white smoker chimney analyzed by Mills and Elderfield (1995) suggesting that, in the near surface, conditions of precipitation or the composition of the fluid may be different from those at depth in the TAG-2 area.

\section{VARIATIONS IN THE REE PATTERNS OF ANHYDRITE RELATIVE TO THE HYDROTHERMAL FLUID}

Given that the concentrations of REEs in seawater are one to four orders of magnitude lower than their concentrations in end-member black smoker hydrothermal fluids (Mitra et al., 1994), the REE patterns of fluids resulting from a mix of the two will resemble the REE pattern of the end-member hydrothermal fluid. Hence, REEs are not good indicators of mixing proportions of hydrothermal fluid and seawater, which are much more readily determined from Sr-isotope data as has been done by Mills et al. (Chap. 10, this volume). The REEs are better suited to investigation of evolution of the fluids as they circulate within the mound and are modified by precipitation and dissolution of various mineral phases.

The most notable feature of all the chondrite-normalized REE patterns from the TAG-1 and TAG-2 areas is their general similarity to each other and to the fluids from which they precipitate. The general REE pattern of the end-member hydrothermal fluids has been attributed to ion exchange during high-temperature alteration of plagioclase in basalts (Klinkhammer et al., 1994), and the similarity of the 

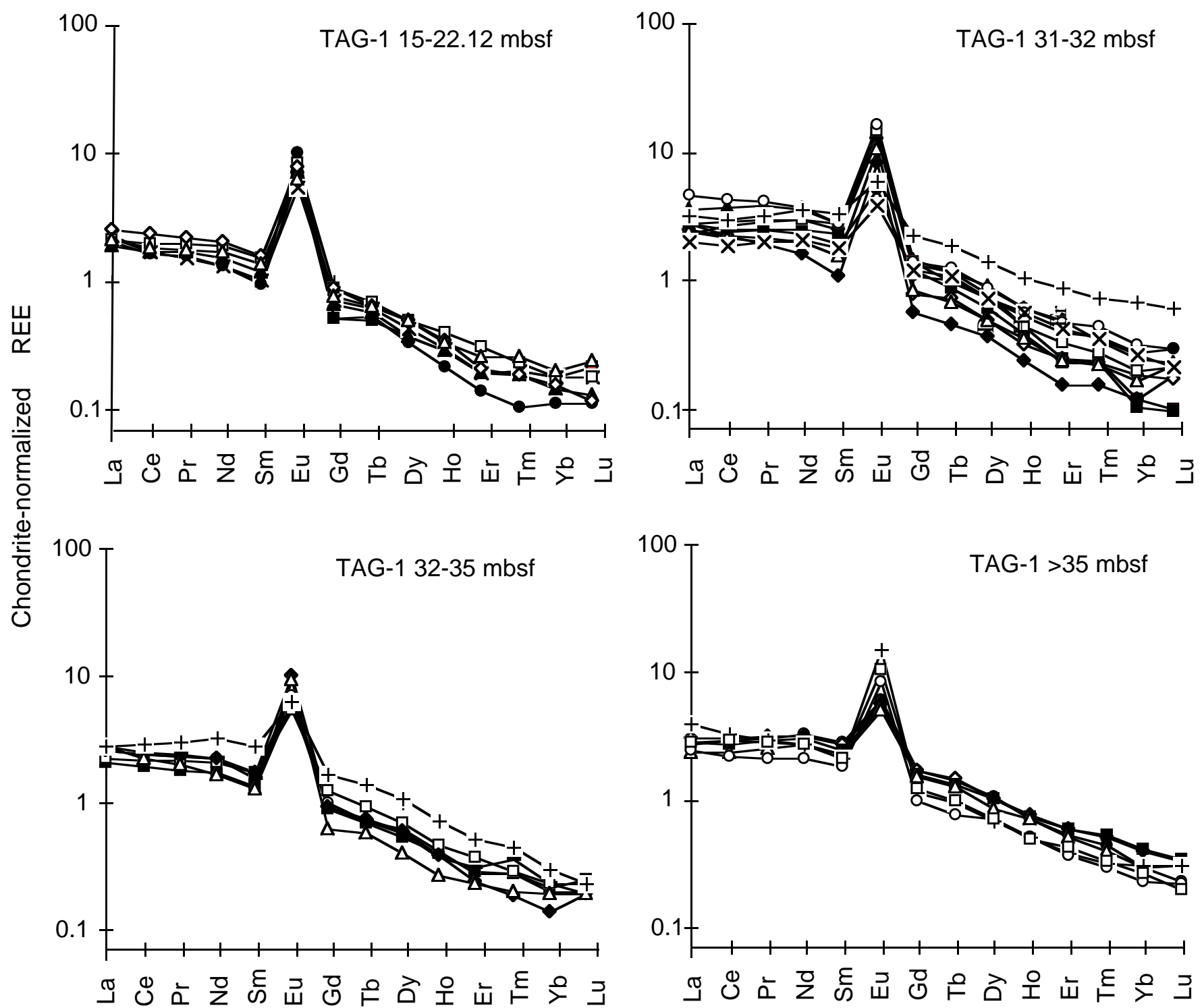

Figure 7. Chondrite-normalized REE patterns for anhydrites from the TAG-1 area. These have been grouped according to depth in order to present the large amount of data.

anhydrite patterns to the fluid patterns has been interpreted to indicate that, with the exception of Eu, the REEs are not fractionated during precipitation of anhydrite from hydrothermal solutions (Mills and Elderfield, 1995).

In order to investigate this further and to examine the behavior of the REEs in the anhydrite relative to the fluid from which it is precipitating, it is useful to normalize the data to the REE concentrations in the fluids using data taken from Mitra et al. (1994). Figure 14 shows the REE patterns for anhydrite from the TAG-1 area normalized to black-smoker fluids and grouped into two different types of REE pattern distinguished on the basis of the behavior of Eu. Table 2 also shows to which group each sample belongs. Fifteen samples distributed throughout the section comprise Group I and includes anhydrite from all textural settings including breccia matrix, cavity fillings, and veins of all three types. They all have relatively flat REE patterns with $\mathrm{Nd} / \mathrm{Yb}$ normalized to the fluid (denoted by $\mathrm{Nd}_{\mathrm{FL}} / \mathrm{Yb}_{\mathrm{FL}}$ ) of $0.8-$ 2.8 that do not vary in any systematic manner. In addition, these samples exhibit only a small or no Eu anomaly ranging from 0.8 to 1.5. This implies that during the precipitation of this type of anhydrite from the circulating fluid, all of the REEs, including Eu, were taken up in proportions that reflected their relative distribution in the fluid. Hence, the precipitation of anhydrite with this type of REE pattern would result only in lowering the concentration of REEs in the fluids, but would not result in any change in the overall REE pattern in the hydrothermal fluid.

The second type of REE pattern that is exhibited by the remaining 17 anhydrite samples from TAG-1 (Group II) is characterized by a distinct negative Eu anomaly ranging from 0.2 to 0.7 relative to the end-member hydrothermal fluid (Fig. 14). These samples are again distributed throughout the section and include breccia matrix and different vein types; hence, the temporal relationship between the two groups cannot be established on the basis of the textural relationships. The negative Eu anomaly implies that during the precipitation of this anhydrite group, Eu is excluded relative to the other REEs. This supports the observation of Mills and Elderfield (1995) that during the evolution of white smoker fluid from black smoker fluid (Fig. 6), there is an increase in fluid $\mathrm{Eu}_{\mathrm{N}} / \mathrm{Eu}^{*}{ }_{\mathrm{N}}$. Hence, anhydrite precipitating from that evolving fluid should also exhibit decreasing REE concen- 


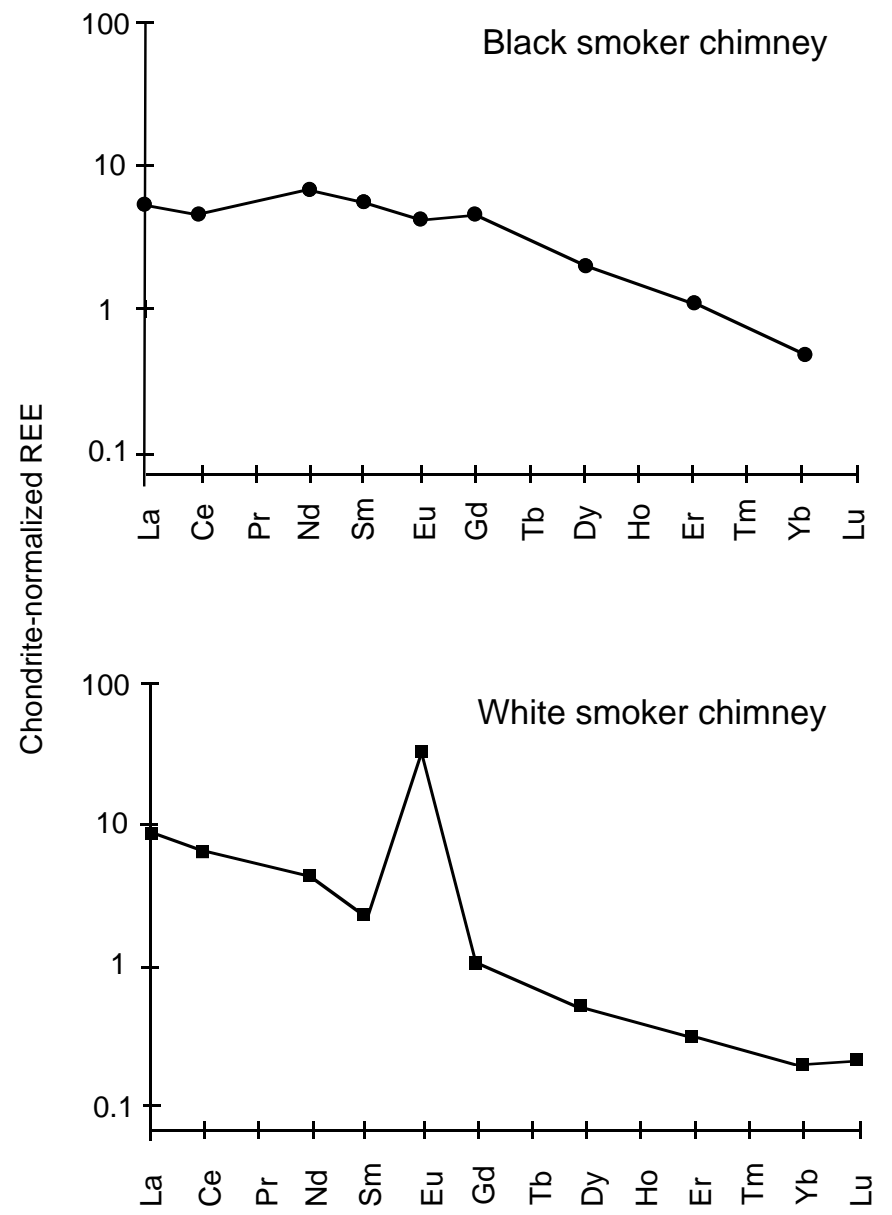

Figure 8. Chondrite-normalized REE patterns for anhydrites from a black smoker chimney (upper diagram) and a white smoker chimney (lower diagram) from TAG presented by Mills and Elderfield (1995).

trations with increasing Eu anomalies (although the size of the Eu anomaly in the chondrite-normalized pattern of anhydrite will be lower than that in the fluid). This relationship is confirmed in Figure 15 , which shows the relation between both the concentration of a single REE (the selection of Gd is arbitrary, but the same result is obtained for other REEs) and the $\sum$ LREE-Eu concentration vs. the $\mathrm{Eu}_{\mathrm{N}} /$ $\mathrm{Eu}^{*}{ }_{\mathrm{N}}$ for each sample.

Mills and Elderfield (1995) also concluded that, with the exception of Eu, the REEs show little or no fractionation during precipitation of anhydrite. However, visual inspection of Figure 6 suggests that changes in the chondrite-normalized REEs during evolution of a black smoker fluid to a white smoker fluid are not proportionate for each element. These variations should therefore also be reflected in the precipitating anhydrite. Although the fluid-normalized La and Ce values are similar for most of the samples in Group II, the HREEs show a wide range in their fluid-normalized values and are generally higher than the corresponding $\mathrm{La}$ and Ce concentration ratios for each sample (Fig. 14). This implies that, during precipitation of anhydrite, the REEs are taken up disproportionately, with the LREEs being excluded relative to the HREEs. Hence, an increase in $\mathrm{La}_{N} / \mathrm{Yb}_{\mathrm{N}}$ in the fluid should be observed. Such a trend is suggested in the black and white smoker fluids of Mitra et al. (1994) in which $\mathrm{La}_{\mathrm{N}} / \mathrm{Yb}_{\mathrm{N}}$ are 11.3 and 36.4, respectively. Although the overall REE pattern of the anhydrite will reflect that of the fluid from which it precipitated, the preferential uptake of the HREE relative to the LREE will result in the anhydrite exhibiting a REE pattern with a lower $\mathrm{La}_{N} / \mathrm{Yb}_{\mathrm{N}}$ than the fluid.

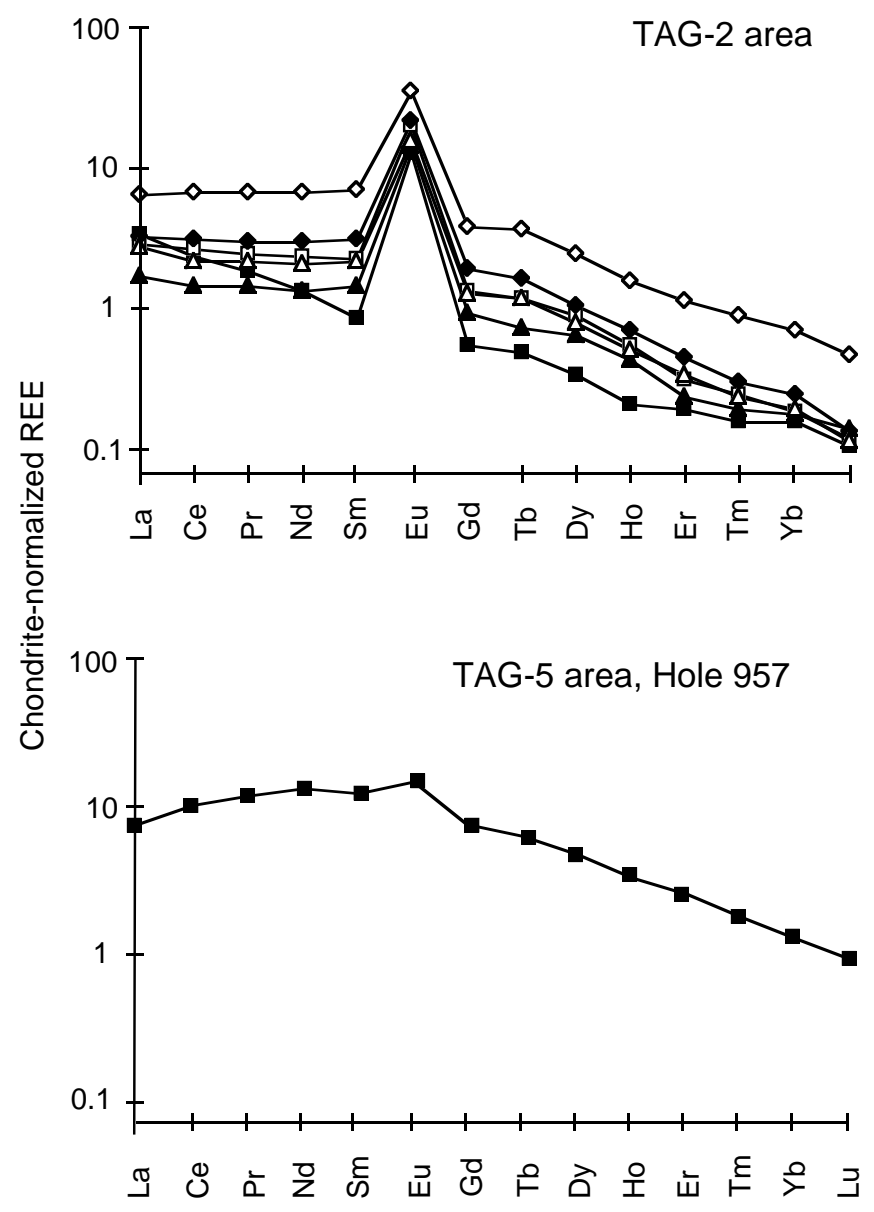

Figure 9. Chondrite-normalized REE patterns for anhydrites from the TAG-2 area (upper diagram) and for one anhydrite sample from the TAG-5 area (lower diagram).

During subsequent mixing and precipitation events, anhydrite will precipitate from fluids with increasing $\mathrm{La}_{\mathrm{N}} / \mathrm{Yb}_{\mathrm{N}}$ (as well as increasing $\left.\mathrm{Eu}_{\mathrm{N}} / \mathrm{Eu}^{*}{ }_{\mathrm{N}}\right)$ and hence, despite the discrimination against the LREE relative to the HREE resulting in anhydrite REE patterns with lower slopes than those of the fluids, the slope of the REE patterns should still increase. This is confirmed in Figure 16 which shows a general trend of increasing Eu anomaly with increasing $\mathrm{La}_{\mathrm{N}} / \mathrm{Yb}_{\mathrm{N}}$ in anhydrite as predicted.

The range in types of anhydrite seen throughout the section at TAG-1 also occurs on a smaller scale within the well-sampled vein at 31.65-32 mbsf. Samples from the upper portion of the complex vein that include the outer, finely banded, crustiform vein, the region of radiating fibers of anhydrite, the anhydrite-filling cavities, and massive granular vein in the interior of the complex vein all fall into Group I, whereas the samples from different growth bands in the crustiform banded vein, together with the lower white vein and the cavity filling near the lower margin of the vein, all belong in Group II. This suggests that there have been at least two generations of anhydrite filling this vein, although their temporal relation is not clear.

The data for anhydrite samples from the TAG-2 area have been normalized to the white smoker fluid compositions (Mitra et al., 1994) and are presented in Figure 17. The patterns are very similar in shape to those of the Group II samples at TAG-1, and show similar covariation between Eu anomaly, LREE enrichment and absolute concentrations of the REEs (Fig. 18). However, the white smoker anhydrites show Eu anomalies that are 2-3 times higher than those at 


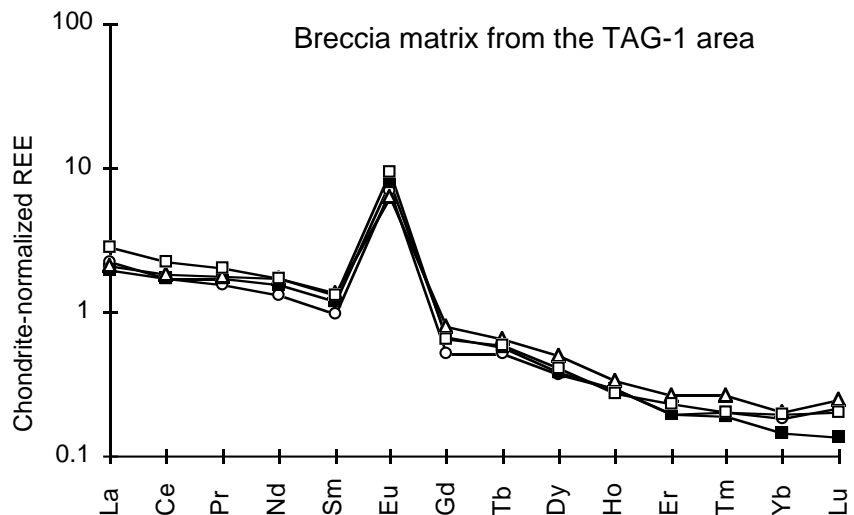

Figure 10. Chondrite-normalized REE patterns for four samples of anhydrite from the matrix of breccias.

similar absolute REE concentrations in the TAG-1 samples. This implies that they precipitated from a hydrothermal fluid characterized by similar REE (excluding Eu) concentrations, but with a higher Eu anomaly than the fluids that precipitated the anhydrite samples at TAG-1. The fluid-normalized REE pattern of anhydrite taken from a white smoker chimney (Mills and Elderfield, 1995) is also shown in Figure 17, and, although flatter, is generally similar.

The REE for the anhydrite sample from the TAG- 5 area has been normalized to white smoker fluid (Fig. 19). Although this area is in closer proximity to the TAG-1 area and the complex of black smokers, fluid inclusion studies reported in this volume by Tivey et al. (1995) indicate that the trapping temperature of this sample was $274^{\circ}-277^{\circ} \mathrm{C}$, which is considerably lower than the temperatures obtained from fluid inclusions in anhydrite in the TAG- 1 area of $338^{\circ}-$ $388^{\circ} \mathrm{C}$. This sample is compared with the anhydrite from the black smoker chimney of Mills and Elderfield (1995; Fig. 19) which had a similar chondrite-normalized REE pattern (Fig. 8). Even though this sample was taken from a black smoker chimney, Mills and Elderfield (1995) calculated a temperature of $235^{\circ} \mathrm{C}$ from fluid mixing proportions derived from Sr-isotope data. Their sample was scraped from the outer wall of the chimney sample and was therefore likely to be anhydrite precipitated at temperatures lower than the discharging black smoker fluid (M. Tivey, pers. comm., 1996). The fluid-normalized REE patterns of these two samples are similar and indicate strong discrimination against Eu. Hence, this type of REE pattern in anhydrites - with the highest absolute REE concentrations and only a slight chondrite-normalized Eu anomaly-was produced at lower temperatures than those from which anhydrite precipitated in the TAG-1 area.

\section{DISCUSSION}

Mixing between hydrothermal fluids (that have been variably altered by precipitation and dissolution of sulfide, sulfate, and oxide phases) and seawater in different proportions within the TAG hydrothermal mound is an extremely dynamic process. Unraveling this process to determine the geochemical evolution of the fluids is very complex, because the composition of the hydrothermal fluid that is mixing with seawater in any part of the circulation system is unknown and cannot be assumed to be that of the end-member black smoker fluid. The results of this study have documented that there are at least three distinct REE patterns produced in anhydrite precipitated during this mixing process. The first (Group I) is characterized by a flat REE pattern normalized to end-member hydrothermal fluid (observed at TAG-1), suggesting that all the REEs are taken up in proportions that directly reflect their distribution in the fluid from which
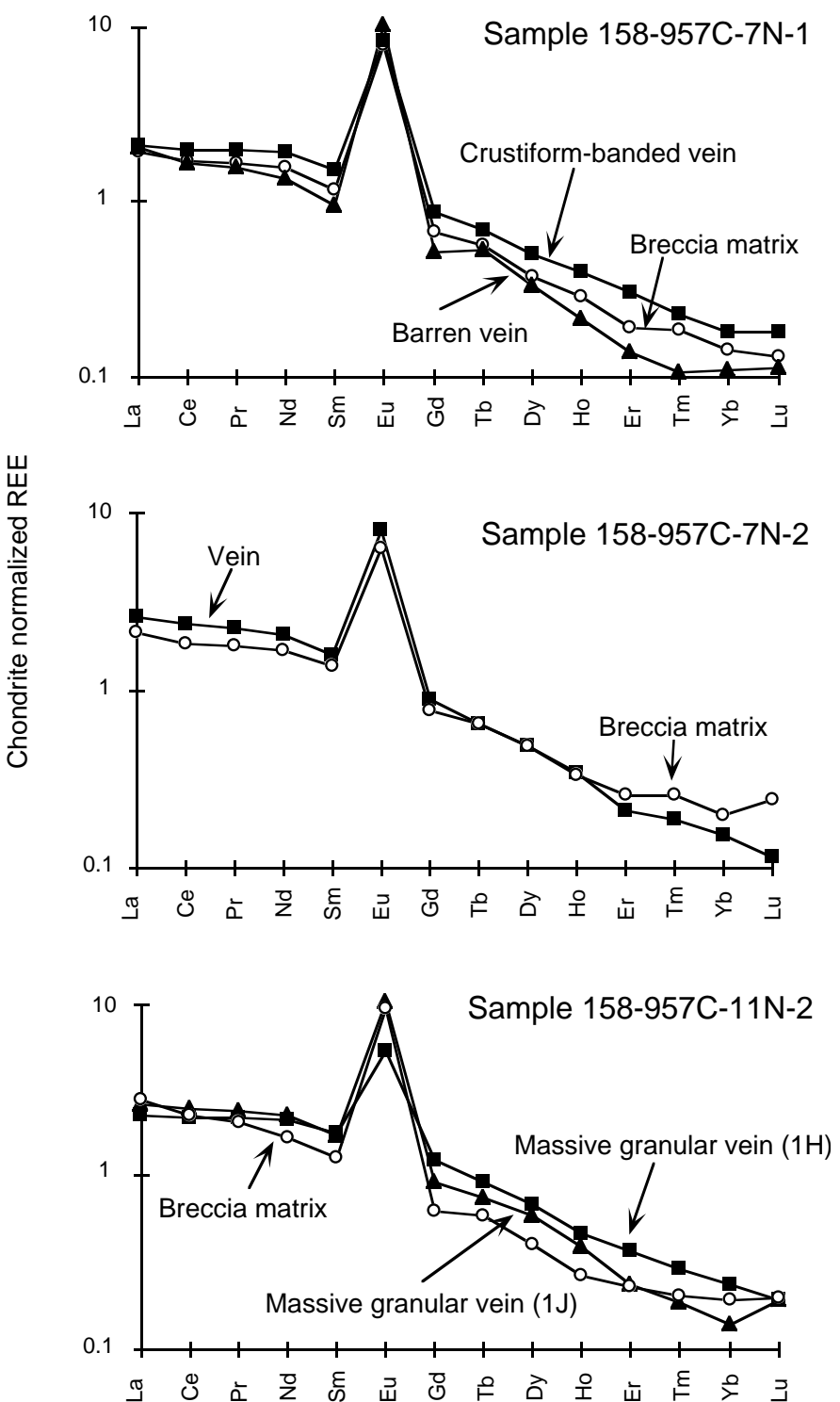

Figure 11. Comparison of chondrite-normalized REE patterns for the anhydrite from veins and breccia matrix within three different samples.

they precipitated. The second is characterized by distinct and increasing Eu anomalies and increasing LREE enrichment with decreasing absolute concentrations of the REEs (observed in Group II at TAG-1 and TAG-2), and the third is characterized by high absolute REE concentrations (excluding Eu) with little or no Eu anomaly and having precipitated at lower temperatures (observed in one near-surface sample at TAG-2 and at TAG-5).

There are a number of factors that can affect the behavior of the REEs in hydrothermal systems, including kinetic effects, complexation in the solution, temperature, and redox chemistry. Non-equilibrium and kinetic effects are extremely difficult to assess, although in this complex, open system they are likely to be important. Fluid inclusion studies on anhydrite have measured trapping temperatures ranging from $338^{\circ}-388^{\circ} \mathrm{C}$ in the TAG-1 area (although none of the TAG-1 samples analyzed in this study were included in the fluid inclusion study), which compares with a temperature of $363^{\circ} \mathrm{C}$ for the discharging hydrothermal fluid (Tivey et al., Chap. 14, this volume). Fluid inclusion trapping temperatures for the TAG-2 area vary from $342^{\circ}-369^{\circ} \mathrm{C}$ (with the same sample from Sample $957 \mathrm{H}-5 \mathrm{~N}-2,55-57$ 
Figure 12. Plot of $\mathrm{Nd}_{\mathrm{N}} / \mathrm{Yb}_{\mathrm{N}}$ and $\mathrm{Eu}_{\mathrm{N}} / \mathrm{Eu}^{*}{ }_{\mathrm{N}}$ values vs. depth for anhydrite samples taken from the $45-\mathrm{cm}$ wide anhydrite vein (Sample 158-957C-11N-1, 120$150 \mathrm{~cm}$ [Pieces 3I, J, K, L]) shown in Figure 4.

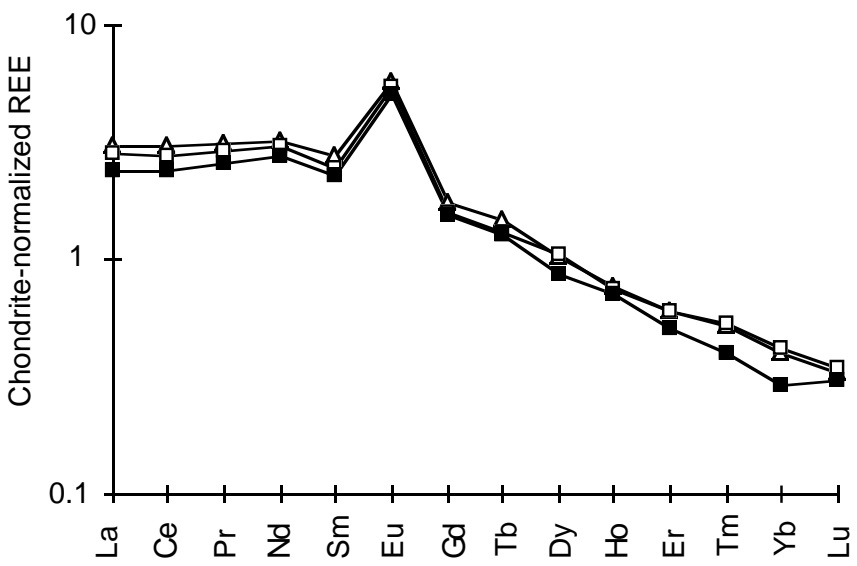

Figure 13. Chondrite-normalized REE patterns for three distinct growth bands within an anhydrite vein at about 35.6 mbsf (Sample 158-957C-12N-1, $82-84 \mathrm{~cm}$, [Piece 8B]).

$\mathrm{cm}$ (Piece 2) as analyzed for REEs giving temperatures of $347^{\circ}-$ $355^{\circ} \mathrm{C}$ ) (Tivey et al., Chap. 14 , this volume). Hence, in general, the temperatures of the fluids from which anhydrite precipitated at depth appear to be in the same range for the TAG-1 and TAG-2 areas. However, analysis of Sample 957H-5N-2, 8-10 cm (Piece 1B) (although not the same sample as analyzed for REEs) from the top of the hole at 9 mbsf gave a cooler temperature of $280^{\circ}-313^{\circ} \mathrm{C}$, which compares more closely with the exiting fluid temperatures of $260^{\circ}-300^{\circ} \mathrm{C}$ (Tivey et al., Chap. 14, this volume). As pointed out in a previous section, this sample contains the highest absolute REE concentrations of any sample from TAG-1 or TAG-2, but a Eu anomaly $\left(\mathrm{Eu}_{\mathrm{N}} / \mathrm{Eu}^{*}{ }_{\mathrm{N}}=\right.$ 6.4) that is smaller than other samples from the TAG-2 area. A similar trend is observed in the sample from the TAG-5 area, which exhibits trapping temperatures of $264^{\circ}-277^{\circ} \mathrm{C}$ (Tivey et al., Chap. 14 , this volume) and yet has the highest absolute REE concentrations of any sample analyzed but a $\mathrm{Eu}_{\mathrm{N}} / \mathrm{Eu}^{*}{ }_{\mathrm{N}}$ value of 1.5 . If the cooler temperatures resulted from mixing of larger proportions of seawater relative to hydrothermal fluid, then the concentrations of the REEs in that fluid should be lower as a result of dilution. In order to maintain high concentrations of the REEs in a mixture of hydrothermal fluid and seawater, it is therefore necessary to have a higher proportion of hydrothermal fluid in the mixture than predicted from the temperatures obtained by simple mixing. This therefore suggests that the hydrothermal fluid had undergone conductive cooling prior to mixing with seawater. An alternative explanation is that REEs had been added to the hydrothermal fluid prior to mixing with seawater by dissolution of anhydrite previously precipitated within the mound.

The temperature, $\mathrm{pH}$, and redox conditions of the hydrothermal fluid are of particular importance to the potential fractionation of $\mathrm{Eu}$ relative to the other REEs. Eu can exist either as a divalent or trivalent ion, with the trivalent ion being closer in size to $\mathrm{Ca}^{2+}$ than the larger divalent ion. At high pressures and temperatures above $250^{\circ} \mathrm{C}$, divalent $\mathrm{Eu}$ should predominate in most natural systems (Sverjensky, 1984; Wood, 1990). Hence, in many of the anhydrite samples analyzed in this study, discrimination against Eu to varying degrees is observed during precipitation of anhydrite and may be the result of the larger ionic radius of the $\mathrm{Eu}^{2+}$ species. As pointed out previously, the size of the Eu anomaly appears to be an indicator of the degree of evolution of the hydrothermal fluid. Hence, the anhydrites from the TAG-2 area have formed from hydrothermal fluids that had previously mixed with seawater and precipitated anhydrite to a greater degree 


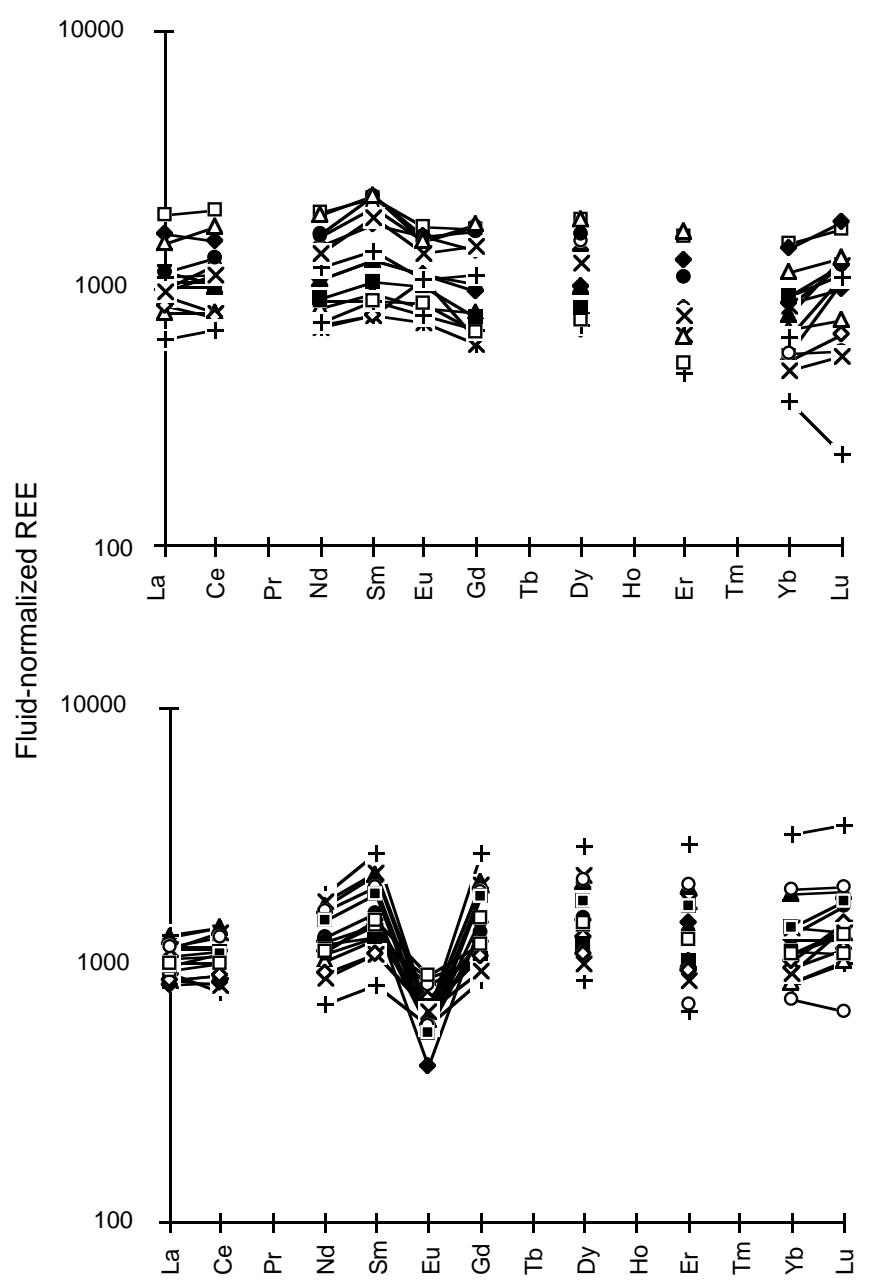

Figure 14. REE compositions of anhydrite from the TAG-1 area normalized to the mean value for end-member black smoker hydrothermal fluids using data from Mitra et al. (1994) and grouped into two different types of REE patterns distinguished on the basis of the behavior of Eu. Samples belonging to Group I (upper diagram) and Group II (lower diagram) are denoted in Table 2.

than the TAG-1 samples, thereby developing stronger Eu anomalies in the fluid. Despite cooling from several mixing episodes, the fluids have remained within the stability field for the $\mathrm{Eu}^{2+}$ species to predominate.

However, at lower temperatures, fluids containing both $\mathrm{Eu}^{2+}$ and $\mathrm{Eu}^{3+}$ (Sverjensky, 1984) may occur. Anhydrite can precipitate from seawater at temperatures of greater than $150^{\circ} \mathrm{C}$ at seafloor pressures. Both conservative mixing and mixing of a hydrothermal fluid with seawater conductively heated before mixing to produce fluids with temperatures of $150^{\circ}-250^{\circ} \mathrm{C}$ will result in $\mathrm{pH}$ values of 5-4.2 and $\log \mathrm{fO}_{2}$ values of -40 to -58 (derived from fig. 3 of Tivey, 1995). Under these conditions, significant activities of both $\mathrm{Eu}^{2+}$ and $\mathrm{Eu}^{3+}$ can occur in the fluids, depending on the oxidation state and the $\mathrm{pH}$ of the solution (from figs. 1 and 3 of Sverjensky, 1984). If it is assumed that the Group I anhydrites were derived from mixing of seawater with an end-member black smoker hydrothermal fluid, then their flat fluid-normalized REE patterns imply that all of the REE, including Eu, were taken up in proportions that reflected their relative distribution in the fluid, rather than discriminating against Eu. A possible explanation for this behavior is that this type of anhydrite was precipitated at lower temperatures and under redox conditions that resulted in $\mathrm{Eu}^{3+}$ being the dominant species, which was not discriminated against during precipitation and

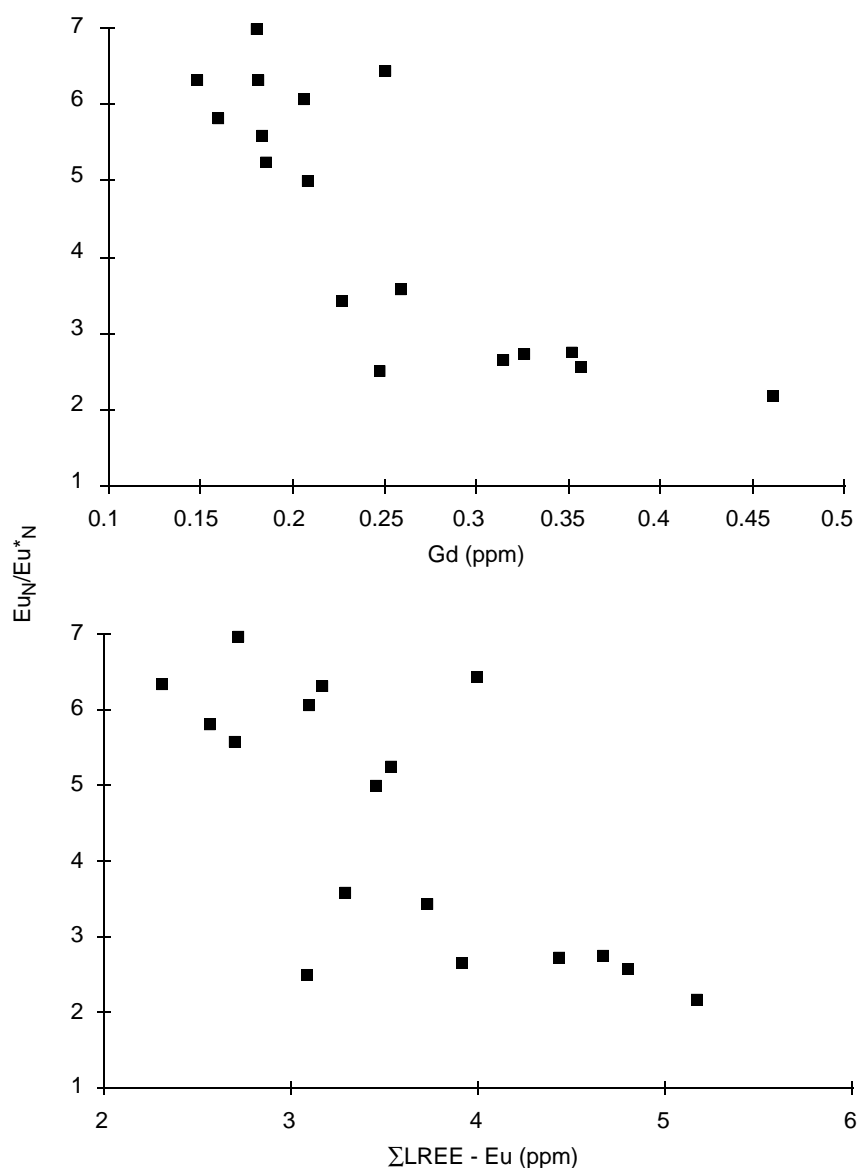

Figure 15. Absolute Gd concentration (upper diagram) and $\sum$ LREE-Eu concentration (lower diagram vs. the $\mathrm{Eu}_{\mathrm{N}} / \mathrm{Eu}^{*}{ }_{\mathrm{N}}$ for the Group II anhydrite samples from the TAG-1 area that show a negative Eu anomaly when normalized to end-member black smoker hydrothermal fluid.

hence there was no fractionation of Eu relative to the other trivalent REEs. Mixing of a hydrothermal fluid with seawater would result in a decrease in temperature and an increase in $\mathrm{fO}_{2}$ in the mix relative to the hydrothermal fluid, both of which would result in more Eu being present as the trivalent ion. However, the fluid inclusion studies suggest that temperatures were generally high in the TAG-1 area, although, because of the lack of paired REE and fluid inclusion measurements, it is not certain that any anhydrites characterized by flat REE patterns were included in the fluid inclusion studies - additional analyses are required to test this model. An alternative possibility that cannot be ruled out is that the Group I anhydrites precipitated from mixing of seawater with a hydrothermal fluid of different composition.

The fractionation of the LREEs from the HREEs was also observed by Mills and Elderfield (1995) in their calculation of the distribution coefficients for La and Ce in anhydrites; however, they attributed this to an artifact caused by the ionic radii values they used. However, the additional analyses presented here suggest that the amounts of La and Ce taken up during anhydrite precipitation are disproportionately low. This is surprising in that the size of the $\mathrm{Ca}^{2+}$ cation is most similar to the lightest REEs and hence should favor replacement by La and Ce. However, Wood (1990) has predicted that the LREEs form complexes with chloride ions in fluids at high temperatures and low $\mathrm{pH}$ values that are more stable than the HREEs. Hence, complexation may play an important role in the observed fractionation of the LREEs from the HREEs during precipitation of anhydrite. 


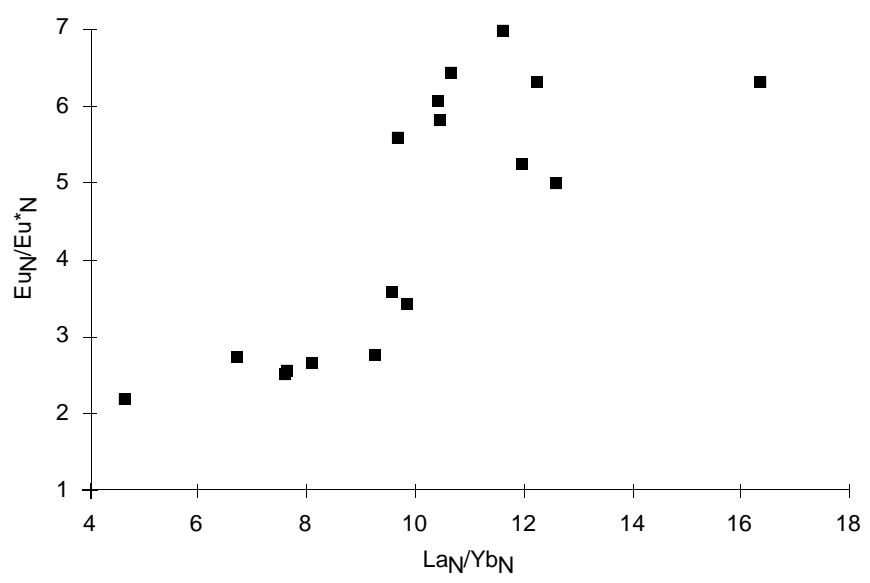

Figure 16. Covariation diagram of $\mathrm{La}_{\mathrm{N}} / \mathrm{Yb}_{\mathrm{N}}$ and $\mathrm{Eu}_{\mathrm{N}} / \mathrm{Eu}^{*}$ for the group of anhydrite samples from the TAG- 1 area that shows a negative Eu anomaly when normalized to end-member black smoker hydrothermal fluid.

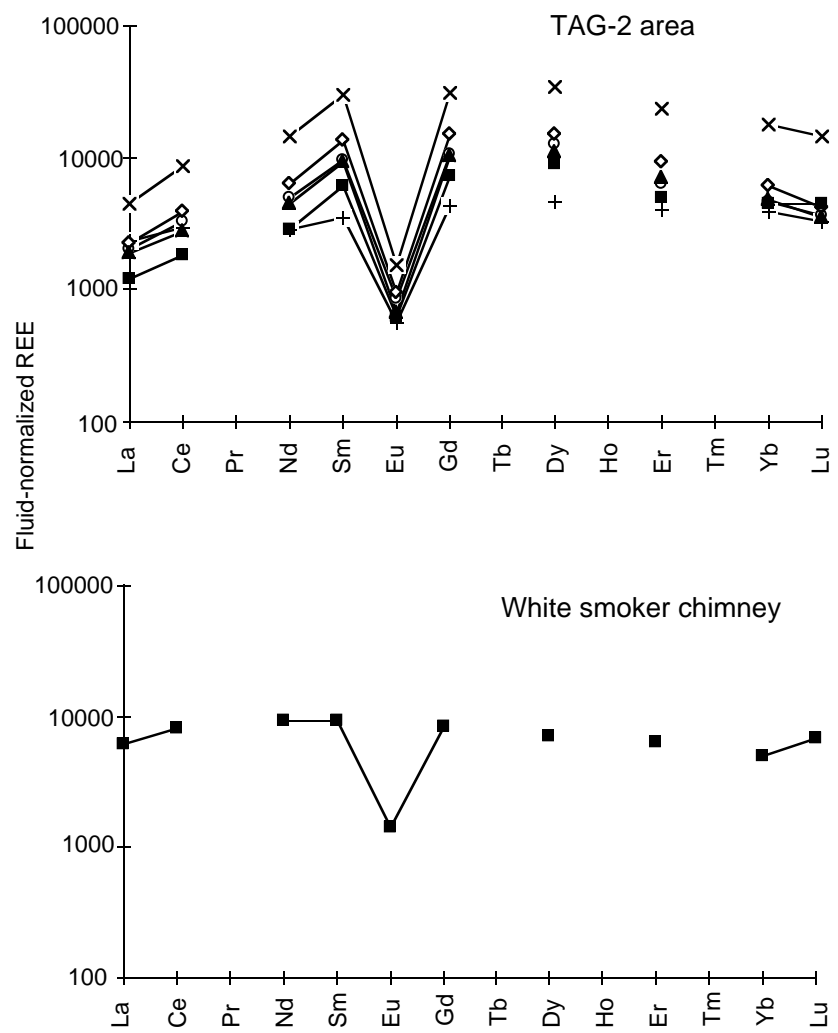

Figure 17. REE compositions of anhydrite from the TAG-2 area normalized to the mean value for white smoker hydrothermal fluids using data from Mitra et al. (1994; upper diagram). Fluid-normalized REE pattern for the white smoker chimney of Mills and Elderfield (1995; lower diagram).

\section{SUMMARY}

Mixing of hydrothermal fluids and seawater has clearly played a critical role in the history of fluid flow and evolution within the mound, and the precipitation and dissolution of anhydrite has a major influence on the style of growth of the mineral deposit. The data presented here have demonstrated that seawater entrainment and mixing extends down to at least $58.68 \mathrm{mbsf}$ and that the mixing process is
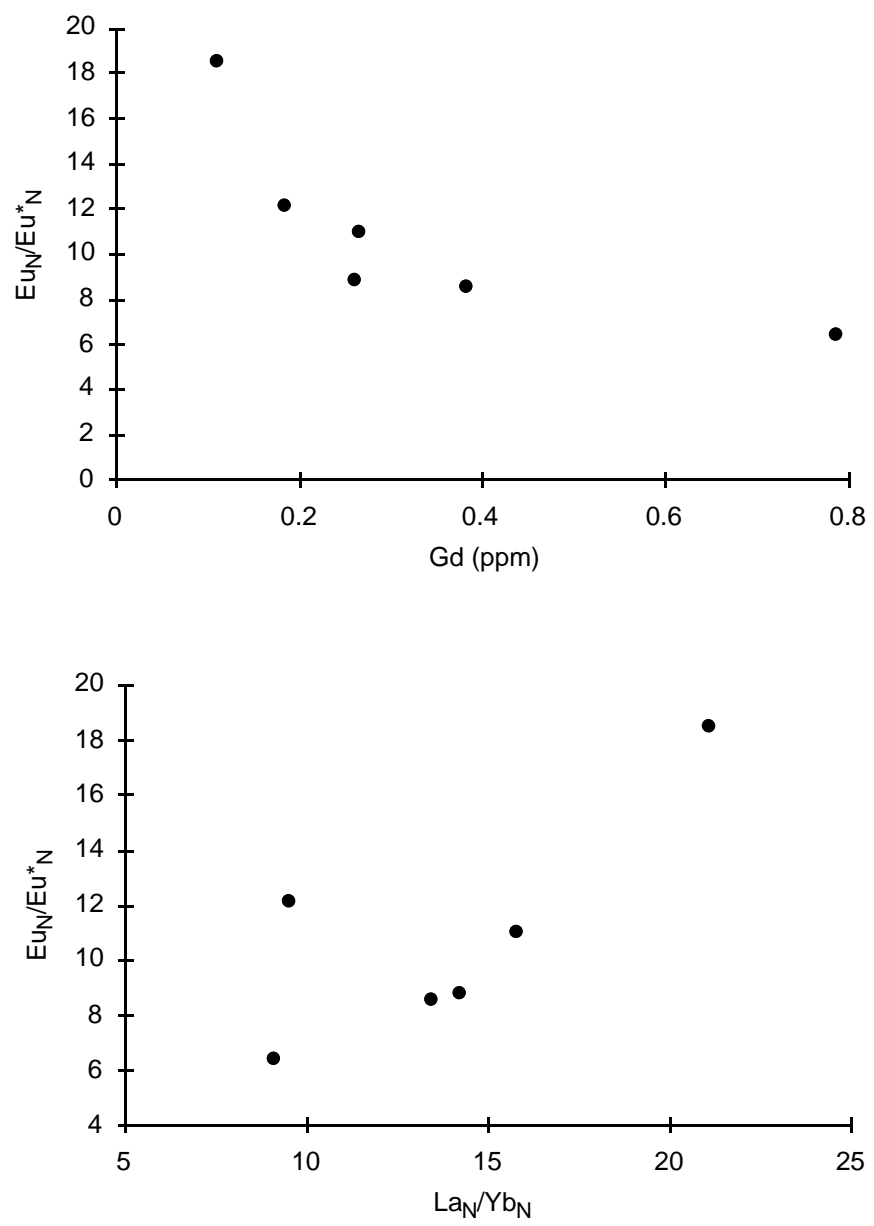

Figure 18. Relations between absolute $\mathrm{Gd}$ concentration, $\mathrm{Eu}_{\mathrm{N}} / \mathrm{Eu}^{*}{ }_{\mathrm{N}}$, and $\mathrm{La}_{\mathrm{N}} / \mathrm{Yb}_{\mathrm{N}}$ for anhydrite samples from the TAG-2 area.

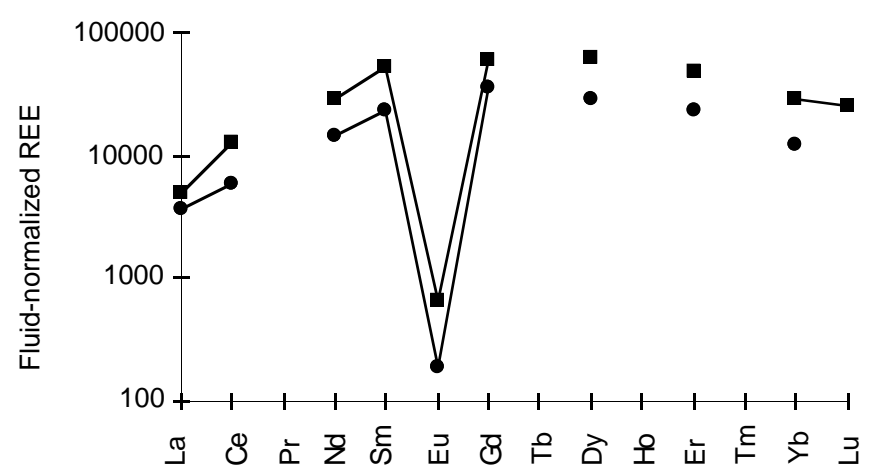

Figure 19. REE composition of the anhydrite sample (squares) from the TAG-5 area normalized to the mean value for white smoker hydrothermal fluids using data from Mitra et al. (1994). Also shown is the fluid-normalized REE pattern for the black smoker chimney (circles) of Mills and Elderfield (1995).

complex and chaotic at all scales, and shows no systematic variations either laterally or vertically on the mound scale, or even within an individual vein. A wide spectrum of mixing, temperature, and redox regimes are possible in the subsurface of the mound, all of which affect the behavior of the REEs. End-member hydrothermal fluids can undergo any number of mixing episodes, either with cold seawater or conductively heated seawater, as well as conductive cooling prior to 
mixing with seawater. During these processes, the changes in temperature and redox conditions will result in differences in the partitioning of the REEs into precipitating anhydrite, thereby resulting in the variability in REE patterns that has been described in this paper. These complex patterns of mixing of seawater with hydrothermal fluids that are continually being modified within an open system make discerning the relations between individual anhydrite samples, and hence the evolution of a hydrothermal fluid within the upflow zone, extremely difficult. Further studies that combine REE determinations with fluid inclusion measurements to assess trapping temperatures, and isotopic studies to investigate mixing proportions, may allow constraints to be placed on the evolution of the hydrothermal fluids.

\section{ACKNOWLEDGMENTS}

This work was supported by USSAC Grant 158-20882 and benefited from discussions with M.K. Tivey. I am indebted to M. Sulanowska for assistance in the preparation of the Figures. C. German and G. Klinkhammer provided helpful and constructive reviews which are very much appreciated. This is WHOI Contribution No. 9377.

\section{REFERENCES}

Campbell, A.C., Palmer, M.R., Klinkhammer, G.P., Bowers, T.S., Edmond, J.M., Lawrence, J.R., Casey, J.F., Thompson, G., Humphris, S., Rona, P.A., and Karson, J.A., 1988. Chemistry of hot springs on the Mid-Atlantic Ridge. Nature, 335:514-519.

Edmond, J.M., Campbell, A.C., Palmer, M.R., German, C.R., Klinkhammer, G.P., Edmonds, H.N., Elderfield, H., Thompson, G., and Rona, P., 1995. Time series studies of vent fluids from the TAG and MARK sites (1986, 1990): Mid-Atlantic Ridge: a new solution chemistry model and a mechanism for $\mathrm{Cu} / \mathrm{Zn}$ zonation in massive sulfide ore bodies. In Parson, L.M., Walker, C.L., and Dixon, D.R. (Eds.), Hydrothermal Vents and Processes. Geol. Soc. Spec. Publ. London, 87:77-86.

Elderfield, H., 1988. The oceanic chemistry of the rare-earth elements. Philos. Trans. R. Soc. London A, 325:105-126.

Elderfield, H., and Greaves, M.J., 1982. The rare earth elements in seawater. Nature, 296:214-219.

Humphris, S.E., Herzig, P.M., Miller, D.J., et al., 1996. Proc. ODP, Init. Repts., 158: College Station, TX (Ocean Drilling Program).

Humphris, S.E., Herzig, P.M., Miller, D.J., Alt, J.C., Becker, K., Brown, D., Brügmann, G., Chiba, H., Fouquet, Y., Gemmell, J.B., Guerin, G., Hannington, M.D., Holm, N.G., Honnorez, J.J., Itturino, G.J., Knott, R., Ludwig, R., Nakamura, K., Petersen, S., Reysenbach, A.-L., Rona, P.A., Smith, S., Sturz, A.A., Tivey, M.K., and Zhao, X., 1995. The internal structure of an active sea-floor massive sulphide deposit. Nature, 377:713-716.

Humphris, S.E., and Kleinrock, M.C., 1996. Detailed morphology of the TAG active hydrothermal mound: insights into its formation and growth, Geophys. Res. Lett., 23:3443-3446.

Kleinrock, M.C., and Humphris, S.E., 1996. Structural controls on seafloor hydrothermal activity at the TAG active mound. Nature, 382:149-153.

Kleinrock, M.C., Humphris, S.E., and the Deep-TAG Team, 1996. Detailed structure and morphology of the TAG active hydrothermal mound and its geotectonic environment. In Humphris, S.E., Herzig, P.M., Miller, D.J., et al., Proc. ODP, Init. Repts., 158: College Station, TX (Ocean Drilling Program), 15-21.

Klinkhammer, G.P., Elderfield, H., Edmond, J.M., and Mitra, A., 1994. Geochemical implications of rare earth elements patterns in hydrother- mal fluids from mid-ocean ridges. Geochim. Cosmochim. Acta, 58:51055113.

Knaack, C., Cornelius, S., and Hooper, P., 1991. Trace element analyses of rocks and minerals by ICP-MS. Washington State Univ., Open File Rep.

Lalou, C., Reyss, J.L., Brichet, E., Arnold, M., Thompson, G., Fouquet, Y., and Rona, P.A., 1993. New age data for Mid-Atlantic Ridge hydrothermal sites: TAG and Snakepit geochronology revisited. J. Geophys. Res., 98:9705-9713.

Lalou, C., Thompson, G., Arnold, M., Brichet, E., Druffel, E., and Rona, P.A., 1990. Geochronology of TAG and Snakepit hydrothermal fields, Mid-Atlantic Ridge: witness to a long and complex hydrothermal history. Earth Planet. Sci. Lett., 97:113-128.

Mills, R.A., and Elderfield, H., 1995. Rare earth element geochemistry of hydrothermal deposits from the active TAG mound, $26^{\circ} \mathrm{N}$ Mid-Atlantic Ridge. Geochim. Cosmochim. Acta, 59:3511-3524.

Mitra, A., Elderfield, H., and Greaves, M.J., 1994. Rare earth element distribution in submarine hydrothermal fluids and plumes from the Mid-Atlantic Ridge. Mar. Chem., 47:217-236.

Rona, P.A., Bogdanov, Y.A., Gurvich, E.G., Rimski-Kursakov, A., Sagalevitch, A.M., Hannington, M.D., and Thompson, G., 1993a. Relict hydrothermal zones in the TAG hydrothermal field, Mid-Atlantic Ridge $26^{\circ} \mathrm{N}$, $45^{\circ}$ W. J. Geophys. Res., 98:9715-9730.

Rona, P.A., Hannington, M.D., Raman, C.V., Thompson, G., Tivey, M.K., Humphris, S.E., Lalou, C., and Petersen, S., 1993b. Active and relict seafloor hydrothermal mineralization at the TAG hydrothermal field, MidAtlantic Ridge. Econ. Geol., 88:1987-2013.

Saunders, A.D., 1984. The rare earth element characteristics of igneous rocks from the ocean basins. In Henderson, P. (Ed.), Rare Earth Element Geochemistry: Amsterdam (Elsevier), 205-236.

Sun, S.-S., and McDonough, W.F., 1989. Chemical and isotopic systematics of oceanic basalts: implications for mantle composition and processes. In Saunders, A.D., and Norry, M.J. (Eds.), Magmatism in the Ocean Basins. Geol. Soc. Spec. Publ. London, 42:313-345.

Sverjensky, D.A.,1984. Europium redox equilibria in aqueous solution. Earth Planet. Sci. Lett., 67:70-78.

Thompson, G., Humphris, S.E., Schroeder, B., Sulanowska, M., and Rona, P.A., 1988. Active vents and massive sulfides at $26^{\circ} \mathrm{N}$ (TAG) and $23^{\circ} \mathrm{N}$ (Snakepit) on the Mid-Atlantic Ridge. Can. Mineral., 26:697-711.

Thompson, G., Mottl, M.J., and Rona, P.A., 1985. Morphology, mineralogy, and chemistry of hydrothermal deposits from the TAG area, $26^{\circ} \mathrm{N}$ MidAtlantic Ridge. Chem. Geol., 49:243-257.

Tivey, M.A., Rona, P.A., and Schouten, H., 1993. Reduced crustal magnetization beneath the active sulfide mound, TAG hydrothermal field, MidAtlantic Ridge $26^{\circ}$ N. Earth Planet. Sci. Lett., 115:101-115.

Tivey, M.K., 1995. Modeling chimney growth and fluid flow. In Humphris, S.E., Zierenberg, R.A., Mullineaux, L.S., and Thomson, R.E. (Eds.), Seafloor Hydrothermal Systems: Physical, Chemical, Biological and Geological Interactions. Am. Geophys. Union, Geophys. Monogr., 91:158177.

Tivey, M.K., Humphris, S.E., Thompson, G., Hannington, M.D., and Rona, P.A., 1995. Deducing patterns of fluid flow and mixing within the TAG active hydrothermal mound using mineralogical and geochemical data. $J$. Geophys. Res., 100:12527-12555.

Wood, S.A., 1990. The aqueous geochemistry of the rare-earth elements and yttrium, 1. Review of available low-temperature data for inorganic complexes and the inorganic REE speciation of natural waters. Chem. Geol., $82: 159-186$.

Date of initial receipt: 3 June 1996

Date of acceptance: 14 January 1997

Ms 158SR-213 